\title{
The first widespread solar energetic particle event observed by Solar Orbiter on 2020 November 29
}

\author{
A. Kollhoff ${ }^{1}$, A. Kouloumvakos ${ }^{2}$, D. Lario ${ }^{3}$, N. Dresing ${ }^{4}$, R. Gómez-Herrero ${ }^{5}$, L. Rodríguez-García ${ }^{5}$, \\ O. E. Malandraki ${ }^{6}$, I. G. Richardson ${ }^{3,7}$, A. Posner $^{8}$, K.-L. Klein ${ }^{9}$, D. Pacheco ${ }^{1}$, A. Klassen ${ }^{1}$, B. Heber ${ }^{1}$, \\ C. M. S. Cohen ${ }^{10}$, T. Laitinen ${ }^{11}$, I. Cernuda ${ }^{5}$, S. Dalla ${ }^{11}$, F. Espinosa Lara $^{5}$, R. Vainio ${ }^{4}$, M. Köberle ${ }^{1}$, R. Kühl ${ }^{1}$, \\ Z. G. Xu ${ }^{1}$, L. Berger ${ }^{1}$, S. Eldrum ${ }^{1}$, M. Brüdern ${ }^{1}$, M. Laurenza ${ }^{12}$, E. J. Kilpua ${ }^{13}$, A. Aran ${ }^{14}$, A. P. Rouillard ${ }^{2}$, \\ R. Bučík ${ }^{15}$, N. Wijsen ${ }^{16}$, J. Pomoell ${ }^{13}$, R. F. Wimmer-Schweingruber ${ }^{1}$, C. Martin ${ }^{1}$, S. I. Böttcher ${ }^{1}$, \\ J. L. Freiherr von Forstner ${ }^{1}$, J.-C. Terasa ${ }^{1}$, S. Boden ${ }^{1}$, S. R. Kulkarni ${ }^{1}$, A. Ravanbakhsh ${ }^{1}$, M. Yedla ${ }^{1}$, N. Janitzek ${ }^{17}$, \\ J. Rodríguez-Pacheco ${ }^{5}$, M. Prieto Mateo ${ }^{5}$, S. Sánchez Prieto ${ }^{5}$, P. Parra Espada ${ }^{5}$, O. Rodríguez Polo ${ }^{5}$, \\ A. Martínez Hellín 5 , F. Carcaboso ${ }^{5}$, G. M. Mason ${ }^{18}$, G. C. Ho ${ }^{18}$, R. C. Allen ${ }^{18}$, G. Bruce Andrews ${ }^{18}$, \\ C. E. Schlemm ${ }^{18}$, H. Seifert ${ }^{18}$, K. Tyagi ${ }^{18}$, W. J. Lees ${ }^{18}$, J. Hayes ${ }^{18}$, S. D. Bale ${ }^{19}$, V. Krupar ${ }^{20,3}$, T. S. Horbury ${ }^{21}$, \\ V. Angelini ${ }^{21}$, V. Evans ${ }^{21}$, H. O'Brien ${ }^{21}$, M. Maksimovic ${ }^{9}$, Yu. V. Khotyaintsev ${ }^{22}$, A. Vecchio ${ }^{23}$, \\ K. Steinvall ${ }^{22,24}$, and E. Asvestari ${ }^{13}$ \\ (Affiliations can be found after the references)
}

Received 30 March 2021 / Accepted 24 May 2021

\begin{abstract}
Context. On 2020 November 29, the first widespread solar energetic particle (SEP) event of solar cycle 25 was observed at four widely separated locations in the inner $(\leqslant 1 \mathrm{AU})$ heliosphere. Relativistic electrons as well as protons with energies $>50 \mathrm{MeV}$ were observed by Solar Orbiter (SolO), Parker Solar Probe, the Solar Terrestrial Relations Observatory (STEREO)-A and multiple near-Earth spacecraft. The SEP event was associated with an M4.4 class X-ray flare and accompanied by a coronal mass ejection and an extreme ultraviolet (EUV) wave as well as a type II radio burst and multiple type III radio bursts.

Aims. We present multi-spacecraft particle observations and place them in context with source observations from remote sensing instruments and discuss how such observations may further our understanding of particle acceleration and transport in this widespread event.

Methods. Velocity dispersion analysis (VDA) and time shift analysis (TSA) were used to infer the particle release times at the Sun. Solar wind plasma and magnetic field measurements were examined to identify structures that influence the properties of the energetic particles such as their intensity. Pitch angle distributions and first-order anisotropies were analyzed in order to characterize the particle propagation in the interplanetary medium.

Results. We find that during the 2020 November 29 SEP event, particles spread over more than $230^{\circ}$ in longitude close to 1 AU. The particle onset delays observed at the different spacecraft are larger as the flare-footpoint angle increases and are consistent with those from previous STEREO observations. Comparing the timing when the EUV wave intersects the estimated magnetic footpoints of each spacecraft with particle release times from TSA and VDA, we conclude that a simple scenario where the particle release is only determined by the EUV wave propagation is unlikely for this event. Observations of anisotropic particle distributions at SolO, Wind, and STEREO-A do not rule out that particles are injected over a wide longitudinal range close to the Sun. However, the low values of the first-order anisotropy observed by near-Earth spacecraft suggest that diffusive propagation processes are likely involved.
\end{abstract}

Key words. Sun: particle emission - Sun: heliosphere - Sun: coronal mass ejections (CMEs) - Sun: flares - interplanetary medium

\section{Introduction}

Solar energetic particle (SEP) events observed by widely separated spacecraft have led to fundamental questions about the underlying processes responsible for the wide spread of energetic particles in the heliosphere. During the Solar Terrestrial Relations Observatory (STEREO) era, different possible explanations for this wide extent of SEPs had been considered (e.g., Rouillard et al. 2012; Gómez-Herrero et al. 2015; Lario et al. 2014, 2017; Zhang et al. 2021, and references therein). The possibility of extended particle sources was discussed even earlier. For example, Cliver et al. (1995) and Torsti et al. (1999) proposed that coronal and interplanetary shocks driven by coronal mass ejections (CMEs) could accelerate and inject particles over large regions in the heliosphere (also see Kouloumvakos et al.
2019). Transport processes are another candidate suggesting that particles originating from even a narrow source could propagate to distant heliolongitude either in the corona or in interplanetary space (e.g., Reinhard \& Wibberenz 1974; Jokipii 1966). Differences in solar wind conditions can produce variations in particle onset delays and intensity-time profiles observed even between locations with small longitudinal separations (e.g., Klassen et al. 2016; Pacheco et al. 2017). Turbulence-induced particle diffusion perpendicular to the magnetic field within the interplanetary medium has been discussed by, for example, Wibberenz \& Cane (2006) and Zhang et al. (2009) and has been supported by multiple modeling efforts (e.g., Dröge et al. 2010; Wang et al. 2012). It has also been shown that the magnetic field line meandering associated with turbulence can give rise to efficient non-diffusive propagation across the average magnetic field 
(Laitinen et al. 2016). Other possible phenomena that may help particle propagation across the field include guiding center drifts (Marsh et al. 2013; Dalla et al. 2013; Wijsen et al. 2020) and propagation along the heliospheric current sheet (Battarbee et al. 2018).

As examples of studies of such widespread events Dresing et al. (2012) concluded, for a widespread SEP event observed on 2010 January 17, that large delays of the observed particle onsets and weak particle anisotropies are consequences of strong perpendicular diffusion in the interplanetary medium (Dröge et al. 2010). In contrast, the clear anisotropies observed during a widespread event on 2011 November 3 disfavor a major role for perpendicular transport in the interplanetary medium and rather suggest that particles spread quickly close to the Sun (Gómez-Herrero et al. 2015). Different delays in the observed particle onset times at different spacecraft have been attributed to either the time the shock needs to intersect with the field lines connected to the individual spacecraft (e.g., Malandraki et al. 2009; Park et al. 2013) or to the time required for interplanetary cross-field diffusion (Dröge et al. 2010).

Multi-spacecraft observations are essential for determining the properties of widespread SEP events. In particular, the STEREO mission with its two spacecraft, equipped with both in situ particle instruments and remote sensing instruments, has made major contributions to understanding these events. With the new era of spacecraft, including Parker Solar Probe (PSP, Fox et al. 2016) and Solar Orbiter (SolO, Müller et al. 2020), we have a great opportunity to clarify the processes leading to the broad particle spread.

In this paper, we illustrate the potential of these new spacecraft by combining observations from SolO, PSP, STEREO-A and multiple near-Earth spacecraft of the first widespread SEP event of solar cycle 25. For the first time, it is possible to compare and combine energetic particle observations from four different locations $\lesssim 1 \mathrm{AU}$ with a wide longitudinal separation.

In Sect. 2, we introduce the different instruments and data sets used in this study. Section 3 provides an overview of the particle observations at the different locations while detailed descriptions of in situ and remote sensing observations are given in Sect. 4. Analysis of the SEP onsets are presented in Sect. 5 together with anisotropy studies. Finally we summarize and discuss the observations in the context of two extreme scenarios in Sect. 6.

\section{Instrumentation}

This study uses data from instruments on board SolO, PSP, STEREO-A, SOlar and Heliospheric Observatory (SOHO), Global Geospace Science Wind satellite (Wind), and Advanced Composition Explorer (ACE) as well as measurements from Earth-based instruments and simulation data. Below we provide a succinct summary of the main data sets used here.

SolO: We utilize energetic particle measurements from the Energetic Particle Detector (EPD, Rodríguez-Pacheco et al. 2020) Supra Thermal Electron and Proton (STEP) instrument, Electron Proton Telescope (EPT), and High Energy Telescope (HET) for electrons in the energy range from a few $\mathrm{keV}$ to a few $\mathrm{MeV}$ and ion measurements obtained from the STEP, EPT, and HET from a few $\mathrm{keV}$ to above $100 \mathrm{MeV}$ nucleon $^{-1}$. While the majority of EPD's data products perform as expected, there are still some unsettled calibration issues. A more detailed description of the instrument performance is given in Wimmer-Schweingruber et al. (2021). For this study we mostly avoided the use of data products with pending calibration issues. An exception are the differential electron and proton fluxes from
HET which are only used for the determination of onset times and the evaluation of electron anisotropies.

Differential ion fluxes from STEP and EPT were calculated by applying proton geometry factors and are labeled as proton fluxes in this work. However, both instruments do not directly distinguish between different ion species (Rodríguez-Pacheco et al. 2020). We note that STEP electron measurements can have large uncertainties if increased ion fluxes were observed in the same energy range at the same time. EPT electron measurements are known to be affected by ions with energies above $400 \mathrm{keV}$ (the possible contribution of ions is well known from similar instruments such as STEREO/SEPT (see Wraase et al. 2018) or ACE/EPAM (Marhavilas et al. 2015).

Magnetic field measurements were obtained from the Solar Orbiter magnetometer (MAG, Horbury et al. 2020). Unfortunately, the Solar Wind Analyser (SWA, Owen et al. 2020) on board SolO was not operational during the event. Instead, solar wind speed and plasma density were obtained by using measurements from the Radio and Plasma Waves (RPW, Maksimovic et al. 2020) instrument. We obtained the electron density $\left(n_{\mathrm{e}}\right)$ from the probe-to-spacecraft potential ( $\left.V_{\mathrm{PSP}}\right)$ measured by RPW. As the spacecraft floating potential is reached when the total current to the spacecraft is zero, that is when the photo-electron emission from the spacecraft is balanced by the plasma electron current, we derived an approximate dependence of $n_{\mathrm{e}}$ on $V_{\text {PSP }}$. Then, we calibrated the electron density by using the electron plasma frequency derived from the high-frequency electric field spectrum (for more details see Khotyaintsev et al. 2021). In order to estimate the SW speed, we applied the deHoffmann-Teller (HT) analysis (Khrabrov \& Sonnerup 1998) to electric and magnetic field data. The method used is described in detail in Steinvall et al. (2021) and is summarized here. The goal of HT analysis is to find the velocity (relative to the spacecraft) of the frame in which the electric field is zero. In the solar wind, where magnetic fluctuations due to current sheets and magnetohydrodynamic (MHD) turbulence are abundant, the HT velocity $\boldsymbol{v}_{\mathrm{HT}}$ is a good estimate of the solar wind velocity. We obtained a series of radial solar wind speeds by repeatedly applying the HT analysis on one-hour intervals of the electric field $\boldsymbol{E}$ and magnetic field $\boldsymbol{B}$ data, moving the centre of the interval in 10-min steps, keeping velocities where $-\left(\boldsymbol{v}_{\mathrm{HT}} \times \boldsymbol{B}\right)_{y}$ was in good agreement with $E_{y}$. Finally, we averaged the velocities over six-hour intervals to reduce noise.

L1-Missions: We utilize particle observations from the Electron Proton Helium INstrument (EPHIN, Müller-Mellin et al. 1995) and from the High Energy Detector (HED) of the Energetic and Relativistic Nuclei and Electron experiment (ERNE, Torsti et al. 1995) on board the SOHO, 3D Plasma and Energetic Particle Investigation (3DP, Lin et al. 1995) on board Wind, and Electron Proton Alpha Monitor (EPAM, Gold et al. 1998) on board ACE. Solar wind plasma and magnetic field observations were taken from the Solar Wind Experiment (SWE, Ogilvie et al. 1995) and Magnetic Field Investigation (MFI, Lepping et al. 1995) on board Wind. Furthermore, we used coronagraph images from the Large Angle and Spectrometric Coronagraph (LASCO)-C2 on board SOHO. While in-depth reviews of the proton and helium fluxes measured by $\mathrm{SOHO} / \mathrm{EPHIN}$ have been performed in recent years (e.g., Kühl \& Heber 2019), the electron measurements are yet to be optimized in the same manner. In particular, the complex energydependent response of the electron channels and the resulting spectral shape dependency of the applied response factor has to be taken into account in order to provide an absolute flux 
number. While this issue is shared with all instruments performing electron measurements in this energy range, it is unfortunately often neglected. To avoid feigning an uncertainty which is yet to be quantified, we preferred to present count rates rather than physical flux numbers. The onset determination of the EPHIN electrons performed in this paper is not affected by this.

STEREO-A: We use particle measurements from the HET (von Rosenvinge et al. 2008), the Solar Electron Proton Telescope (SEPT, Müller-Mellin et al. 2008), and the Suprathermal Electron Telescope (STE, Lin et al. 2008), radio observations from the Radio and Plasma Wave Investigation on the STEREO Mission (SWAVES, Bougeret et al. 2008), EUV images from the Sun Earth Connection Coronal and Heliospheric Investigation (SECCHI) - The Extreme-Ultraviolet-Imager (EUVI) as well as coronagraph images from SECCHI - The Coronagraph COR2 (COR2; Howard et al. 2008). As for EPT, SEPT ion fluxes are calculated by applying proton geometry factors and are labeled as proton fluxes here. We note that SEPT electron measurements are affected by ions with energies above $400 \mathrm{keV}$ (Wraase et al. 2018). Magnetic field and solar wind plasma measurements were obtained from the Magnetic Field Experiment (MAG) and the Plasma and Suprathermal Ion Composition experiment (PLASTIC, Galvin et al. 2008). We note that for this study we use preliminary level 2 data from PLASTIC as no further evaluated data was available for the time period considered here.

PSP: Particle observations by PSP were provided by the Integrated Science Investigation of the Sun (IS $\odot$ IS, McComas et al. 2016). IS $\odot$ IS is made up of two Energetic Particle Instruments (EPI), covering the low (EPI-Lo) and high (EPI-Hi) portions of the energetic particle distribution. EPI-Hi is further comprised of three telescopes: two Low Energy Telescopes (one double ended with apertures labeled LETA and LETB, the other single ended and labeled LETC) and a double ended High Energy Telescope (with apertures called HETA and HETB). The data shown in Fig. 1 are from EPI-Lo and HET (averaged over HETA and HETB). It should be noted that complete calibration of the instruments' responses to electrons have not been completed, so currently only count rates are available.

Ground-based and Earth orbit observations: We use radio observations from the Compound Astronomical Lowcost Lowfrequency Instrument for Spectroscopy and Transportable Observatory (CALLISTO) instruments in Glasgow, Heiterswil (Switzerland) and Trieste in the $45-81 \mathrm{MHz}$ range (Benz et al. 2005) and from the Observations Radio pour Fedome et l'Etude des Eruptions Solaires (ORFEES) in Nançay in the range 144$1000 \mathrm{MHz}$. In addition, we utilize images from the Solar Ultraviolet Imager (SUVI, Vasudevan et al. 2019) of the Geostationary Operational Environmental Satellite (GOES) and Atmospheric Imaging Assembly (AIA, Lemen et al. 2011) on board the Solar Dynamics Observatory (SDO).

Solar Wind Simulations: We use the Wang-Sheeley-Arge (WSA)-ENLIL+Cone model (ENLIL model, Odstrčil et al. 1996; Arge \& Pizzo 2000; Odstrcil 2003; Arge et al. 2004) to model the changing solar wind conditions at the time of the SEP event, including propagation of the associated CME, and to derive the interplanetary magnetic field (IMF lines as an alternative to using nominal Parker spiral field lines. This is a global 3D MHD model ${ }^{1}$ that generates a time-dependent background characterization of the heliosphere outside of $21.5 R_{\odot}$. The simulation uses a time-dependent sequence of daily-updated Global Oscillations Network Group (GONG) magnetograms as a base,

\footnotetext{
1 https://ccmc.gsfc.nasa.gov/models/modelinfo.php? model=ENLIL\%20with\%20Cone\%20Model
}
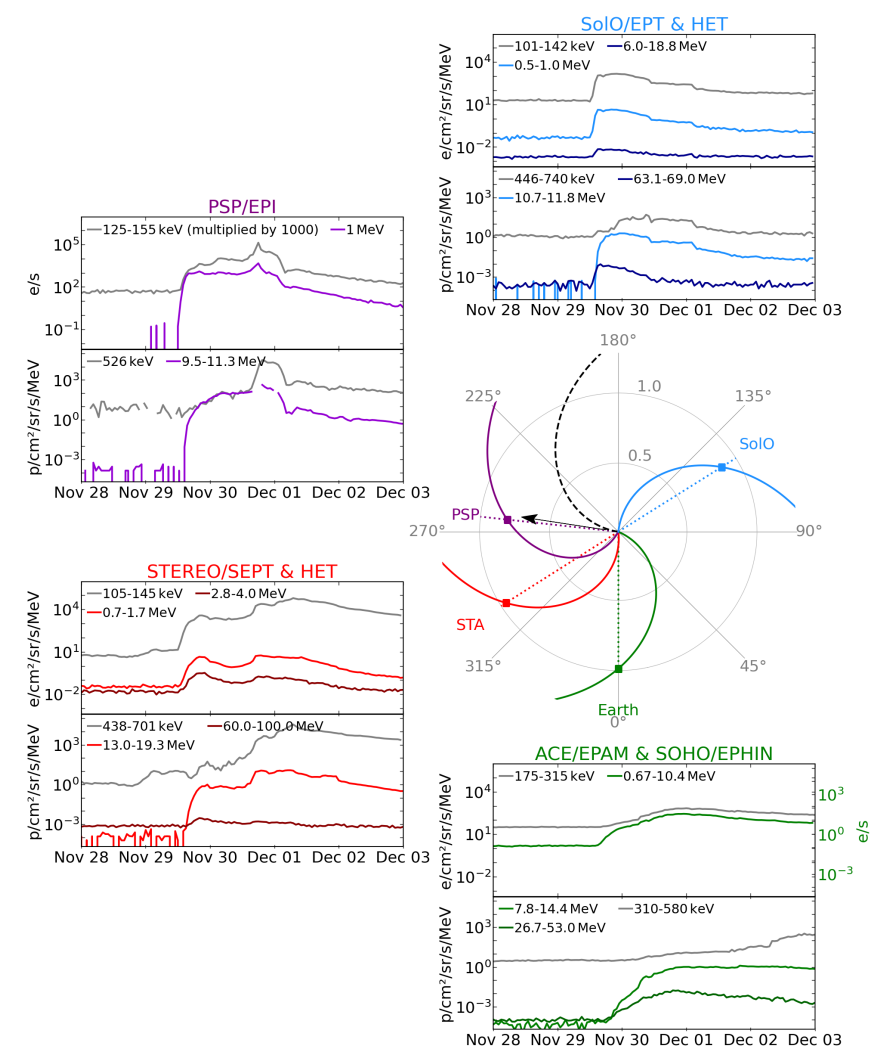

Fig. 1. Overview of the 2020 November 29 SEP event: middle right panel shows the orbital locations of PSP (magenta point), SolO (blue point), STEREO-A (red point), and near-Earth spacecraft (green point) as seen from the north ecliptic. Nominal interplanetary magnetic field (IMF) lines connecting each spacecraft with the Sun considering the solar wind speeds listed in Table 1 are shown in corresponding colors. The black arrow indicates the location of the active region associated with the event (E98 seen from the Earth). Surrounding panels illustrate hourly-averaged proton fluxes (lower panels) and electron fluxes (upper panels) observed by the different spacecraft.

into which high density structures are injected to model solar wind disturbances. We use the graduated cylindrical shell (GCS) model (Thernisien et al. 2006; Thernisien 2011) to reconstruct the CMEs injected in the ENLIL model (see details in NievesChinchilla et al., in prep.). As well as modeling the heliospheric structure, ENLIL provides the magnetic footpoints and the IMF lines passing through the different spacecraft, so the magnetic field lines length can be estimated. The data derived from the modeled IMF lines at the SEP onset time is summarized in Table 1. The input parameters and results of the model are available on the Community Coordinated Modeling Center (CCMC) website ${ }^{2}$.

\section{Energetic particle increase on $\mathbf{2 0 2 0}$ November 29}

Figure 1 shows an overview of the particle observations from 2020 November 28 to 2020 December 3 collected by SolO (top right panel), PSP (top left panel), STEREO-A (bottom left panel), and by near-Earth spacecraft (bottom right panel). Each panel shows intensity-time profiles of electrons (upper portion of the panels) and protons (lower portion of the panels) as observed by the EPI-Lo (gray) and EPI-Hi (magenta)

\footnotetext{
2 https://ccmc.gsfc.nasa.gov/database_SH/Laura_ Rodriguez-Garcia_031321_SH_1.php
} 
Table 1. Spacecraft locations, magnetic field footpoints, IMF path length $(L)$ and longitudinal separation ( $\Delta$ Lon.) from the flare location (HGC: $249^{\circ}$ longitude; $-23^{\circ}$ latitude).

\begin{tabular}{|c|c|c|c|c|c|c|c|c|c|c|c|c|}
\hline \multicolumn{4}{|c|}{ Location $^{(a)}$} & \multicolumn{9}{|c|}{ Magnetic field footpoints ${ }^{(a)}$} \\
\hline \multirow[b]{2}{*}{ Spacecraft/Body } & \multirow[b]{2}{*}{$r(\mathrm{AU})$} & \multirow[b]{2}{*}{ Lon. } & \multirow[b]{2}{*}{ Lat. } & \multicolumn{5}{|c|}{ Parker spiral } & \multicolumn{4}{|c|}{ ENLIL $^{(b)}$} \\
\hline & & & & $\operatorname{Vsw}\left(\mathrm{km} \mathrm{s}^{-1}\right)$ & Lon. & Lat. & $\Delta$ Lon. & $L(\mathrm{AU})$ & Lon. & Lat. & $\Delta$ Lon. & $L(\mathrm{AU})$ \\
\hline STEREO-A & 0.96 & $290^{\circ}$ & $7^{\circ}$ & 361 & $355^{\circ}$ & $7^{\circ}$ & $106^{\circ}$ & 1.15 & $344^{\circ}$ & $6^{\circ}$ & $95^{\circ}$ & 1.16 \\
\hline Earth & 0.99 & $348^{\circ}$ & $1^{\circ}$ & 358 & $55^{\circ}$ & $1^{\circ}$ & $166^{\circ}$ & 1.18 & $44^{\circ}$ & $1^{\circ}$ & $155^{\circ}$ & 1.24 \\
\hline PSP & 0.81 & $251^{\circ}$ & $4^{\circ}$ & $295^{(b)}$ & $319^{\circ}$ & $4^{\circ}$ & $69^{\circ}$ & 0.98 & $298^{\circ}$ & $4^{\circ}$ & $49^{\circ}$ & 0.94 \\
\hline Solar orbiter & 0.88 & $110^{\circ}$ & $-5^{\circ}$ & $417^{(b)}$ & $162^{\circ}$ & $-5^{\circ}$ & $-88^{\circ}$ & 1.0 & $145^{\circ}$ & $-6^{\circ}$ & $-104^{\circ}$ & 0.94 \\
\hline
\end{tabular}

Notes. ${ }^{(a)}$ Coordinates are given in the Carrington Heliographic (HGC) system. ${ }^{(b)}$ ENLIL values are taken from simulation on $29 / 11 / 2020$ at 13:00 UT. Path length calculated for ENLIL magnetic field lines assume a radial extension from $21.5 R_{\odot}$ to $1 R_{\odot}$.

detectors of IS $\odot$ IS on board PSP, the EPT (gray) and HET (blue) on board SolO, the SEPT (gray) and HET (red) on board STEREO-A, EPAM (gray) on board ACE, and Electron Proton Helium INstrument (EPHIN) (green) on board SOHO.

The view of the ecliptic plane from solar north (middle right panel of Fig. 1) illustrates the spacecraft locations on November 29 at 13:00 UT close to the time when the onset of the large SEP event was observed. These spacecraft covered a longitude span of $\sim 238^{\circ}$ and clearly observed intensity increases of protons at energies $>50 \mathrm{MeV}$ and electrons at energies $>1 \mathrm{MeV}$.

In the middle right panel, we also show nominal Parker spiral IMF lines connecting each spacecraft with the Sun. These lines are calculated using the one hour averaged solar wind speed measured near the event onset time by the STEREO/PLASTIC and the WIND/SWE. Because PSP solar wind data are not available at the time of writing, and the solar wind analyzer on SolO/SWA was not operational, speeds obtained from ENLIL simulation are used instead.

The SEP event was associated with an M4.4 soft X-ray flare that occurred at NOAA active region (AR) 12790, located just behind the east limb as seen from Earth. The flare started at 12:34 UT and peaked at 13:11 UT. STEREO-A/EUVI images at 13:00 UT show that the flare was located at E98S23 (in Stonyhurst Heliographic (HGS) coordinates). The black arrow in the middle right panel of Fig. 1 indicates the longitudinal location of this solar flare. The locations of the different spacecraft and their magnetic footpoints as well as the longitudinal separations between the footpoints and the flare location are given in Table 1. Additionally, as further discussed in Sect. 4.2, the SEP event was associated with the eruption of a fast and relatively wide CME, an EUV-wave expanding in the low corona, and a white-light shock wave observed higher in the corona seen by STEREO$\mathrm{A} / \mathrm{COR} 2$ and $\mathrm{SOHO} / \mathrm{LASCO}-\mathrm{C} 2$.

\section{Observations}

\subsection{In situ observations}

Figure 2 shows, from top to bottom, near-relativistic electron intensities, ion intensities, magnetic field magnitude, magnetic field elevation and azimuth angles in the spacecraft centered Radial-Tangential-Normal (RTN) coordinate system, and the solar wind proton speed and density as measured by, from left to right, SolO, STEREO-A, and near-Earth spacecraft at L1.

The electron and high-energy $(\gtrsim 12 \mathrm{MeV})$ proton intensity profiles at SolO show a rapid rise shortly after the parent solar eruption (indicated by the vertical arrow in the top panels of Fig. 2) followed by a gradual decay. Such intensity-time profiles are typical of SEP events that originate from western longitudes relative to the observing spacecraft (Cane et al. 1988). At about $\sim 11 \mathrm{UT}$ on November 30 , electron and $\approx 500 \mathrm{keV}$ ion intensities drop abruptly in association with the passage of an enhanced magnetic field structure. This structure is most likely a corotating interaction region (CIR) formed by the interaction of a faster solar wind stream with the preceding slower solar wind (indicated by CIR in the third panel of the left column in Fig. 2). Unfortunately, the limited Radio and Plasma Waves (RPW) data cannot confirm the expected transition in solar wind speed at SolO, but this was evident when the same CIR passed Earth on November 21 (not shown here). The abrupt changes in the particle intensities occur in the vicinity of magnetic field sector boundary crossings and the stream interface (indicated by the first dashed vertical line in the left panels of Fig. 2 coinciding with a drop in the solar wind density within the CIR). The ion intensities at even lower energies $(\leqslant 500 \mathrm{keV})$ either plateau at their maximum intensities or keep increasing after the passage of this CIR structure. Such a low-energy ion intensity enhancement suggests that either the CIR affected the transport of low-energy SEPs injected at the time of the solar eruption or that these low-energy ions were locally accelerated in the CIR.

Another abrupt change in the particle intensities at SolO occurs early on December 1 (second dashed vertical line in the left panels of Fig. 2) when intensities decrease, except for the lowest energy $(\$ 20 \mathrm{keV})$ protons, which show a large increase. This intensity change also appears to be associated with an abrupt plasma density decrease, but the absence of a magnetic field enhancement makes it unclear whether this was also a stream interface within a CIR. However, the limited speed estimates from RPW on December 2 appear to be consistent with the presence of a higher speed stream following a CIR. The lower energy ions were sufficiently intense that they contaminated the electron observations made by SolO/STEP during the periods indicated by the gray traces on the top left panel of Fig. 2 .

Another point to note is the presence of multiple sector boundary crossings for around a day ahead of the CIR on November 30 , including during the early stages of the SEP event, indicating that $\mathrm{SolO}$ was close to the heliospheric plasma sheet at this time, with possible implications for SEP transport. Thus, the SolO observations suggest that the arrival of energetic particles at the spacecraft depends on the region where they are accelerated and the influence of solar wind structures on their transport in the inner heliosphere, and that both of these factors depend on the energy of the particles (e.g., Wijsen et al. 2021).

Particle intensities at STEREO-A (center column of Fig. 2) also increase shortly after the occurrence of the solar eruption 

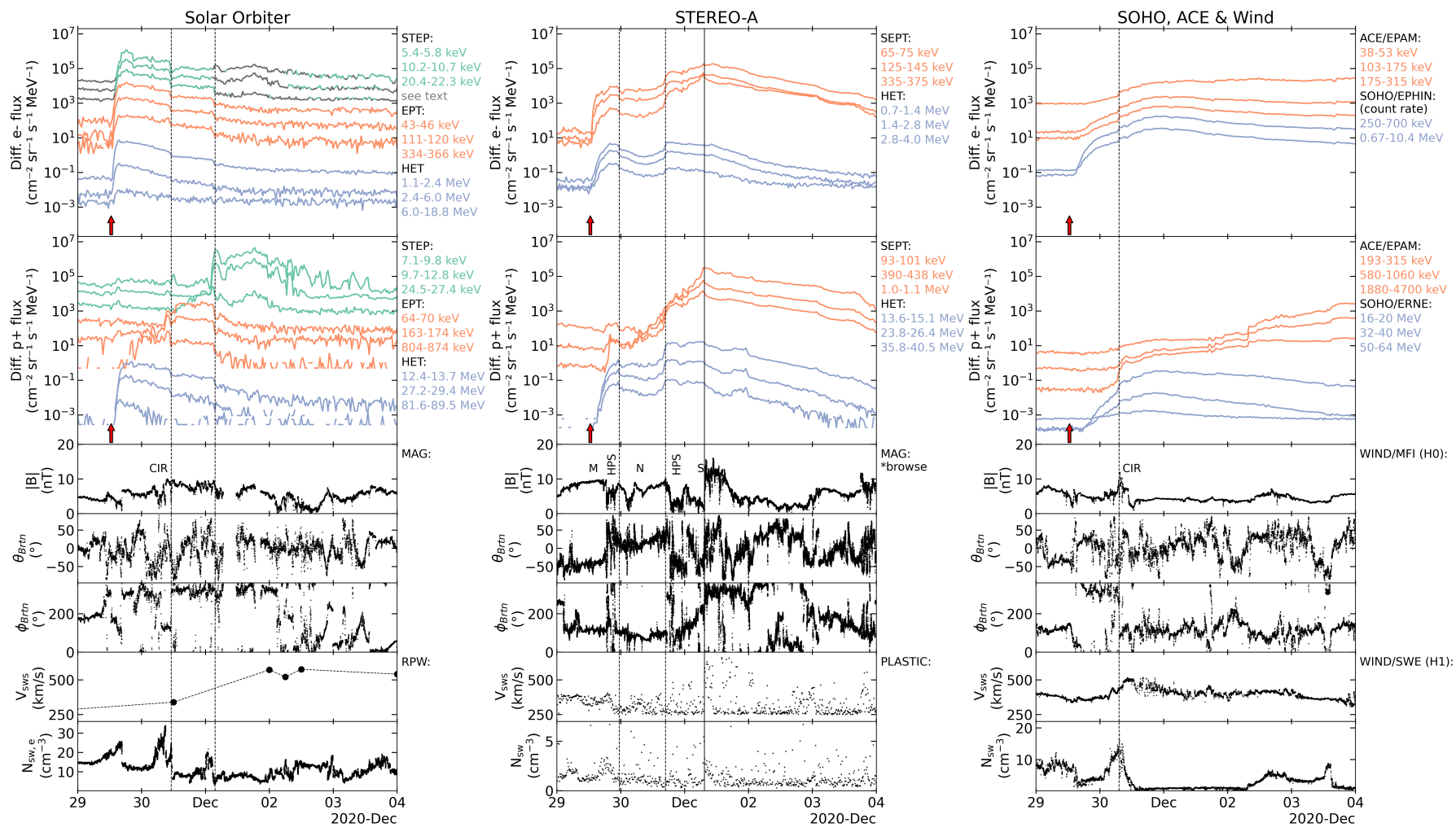

Fig. 2. Time profiles of in situ observations from SolO (left panel), STEREO-A (central panel), and SOHO, ACE at L1 (right panel). Shown from top to bottom are electron fluxes at multiple energies, proton fluxes at multiple energies, magnetic field magnitude, elevation and azimuth angles of the magnetic field vector in RTN coordinates, solar wind speed, and solar wind density. Vertical dashed lines indicate times of abrupt changes in particle intensity. The solid line at STEREO-A indicates passage of a probable shock. Gray lines in the STEP data indicate time periods without clear electron measurements and should be interpreted as an upper limit for the electron flux.

and decay more slowly, but their profiles are more irregular than at SolO. The onset of the event occurs just before the trailing edge of an enhanced, inward-polarity magnetic field structure indicated by "M"; note also that the low-energy $(\lesssim 2 \mathrm{MeV})$ ion intensities are already elevated prior to the event onset. Uncertainty in the STEREO-A/PLASTIC preliminary solar wind plasma data used in this figure does not allow a complete characterization of structure $\mathrm{M}$, but the observation of a similar structure by PSP on November 27 and at L1 on December 4 (not shown here) suggests that this was a corotating structure. Intervals of depressed magnetic field intensity including multiple sector boundaries that are likely to be encounters with the heliospheric plasma sheet (HPS) are also prominent features of the solar wind at STEREO-A during the SEP event.

After several magnetic field sector boundary crossings (first dashed line in the center column in Fig. 2), the electron and high-energy $(\gtrsim 13 \mathrm{MeV})$ proton intensities decrease during the passage of a region of predominantly enhanced $(\sim 10 \mathrm{nT})$ magnetic field with a northward orientation, indicated by " $N$ " in the middle column of Fig. 2. The second vertical dashed line in the center column of Fig. 2 indicates the end of this structure and re-entry into the HPS. The intensities of both electrons and protons at all energies abruptly increase at this time, consistent with a spatial modulation due to the local solar wind structure. The low-energy $(\lesssim 4 \mathrm{MeV})$ ion intensities peak with the arrival of a probable interplanetary shock (to be confirmed when the final plasma data become available) at 07:25 UT on December 1 that presumably was driven by the halo CME on 29 November (the average shock transit speed from the Sun to STEREO-A is $\sim 930 \mathrm{~km} \mathrm{~s}^{-1}$ ). Thus, the STEREO-A observations also show the clear influence of local solar wind structures on the SEP intensities.

Particle intensities observed by near-Earth spacecraft (right panel in Fig. 2) show a more gradual increase with an onset that is substantially delayed with respect to the other spacecraft (see Sect. 5.1). Similar to the rising phase of the event at SolO, a CIR (indicated by "CIR") ahead of a modest high-speed stream affects the intensity profiles. In particular, an abrupt intensity increase during the rising phase of the SEP event is associated with the passage of a true sector boundary crossing (indicated by the vertical dashed line in the right column of Fig. 2), as verified by a reversal of Wind 3DP suprathermal electron flow relative to the magnetic field direction (not shown here). A further abrupt increase in the low energy proton intensity on December 2 is associated with an increase in density that may indicate the trailing edge of the stream.

Thus, Fig. 2 shows that the SEP intensity-time profiles observed by SolO, STEREO-A, and near-Earth spacecraft were also affected by the passage of local solar wind structures. In most cases, these appear to be corotating features but their possible observation by the different spacecraft, and potential influence of transient structures, requires further analyses of the solar wind data and modeling of these structures in the inner heliosphere.

The SEP intensity-profiles observed at PSP (top left panel Fig. 1) are studied in detail by Cohen et al. (2021). Although there appears to be a small shock and possibly an ICME observed by PSP prior to the Nov 29 event (due to the slower CME launched late on November 26), there is no corresponding $\mathrm{SEP}$ signature. The $10 \mathrm{MeV}$ proton intensity and $1 \mathrm{MeV}$ electron 

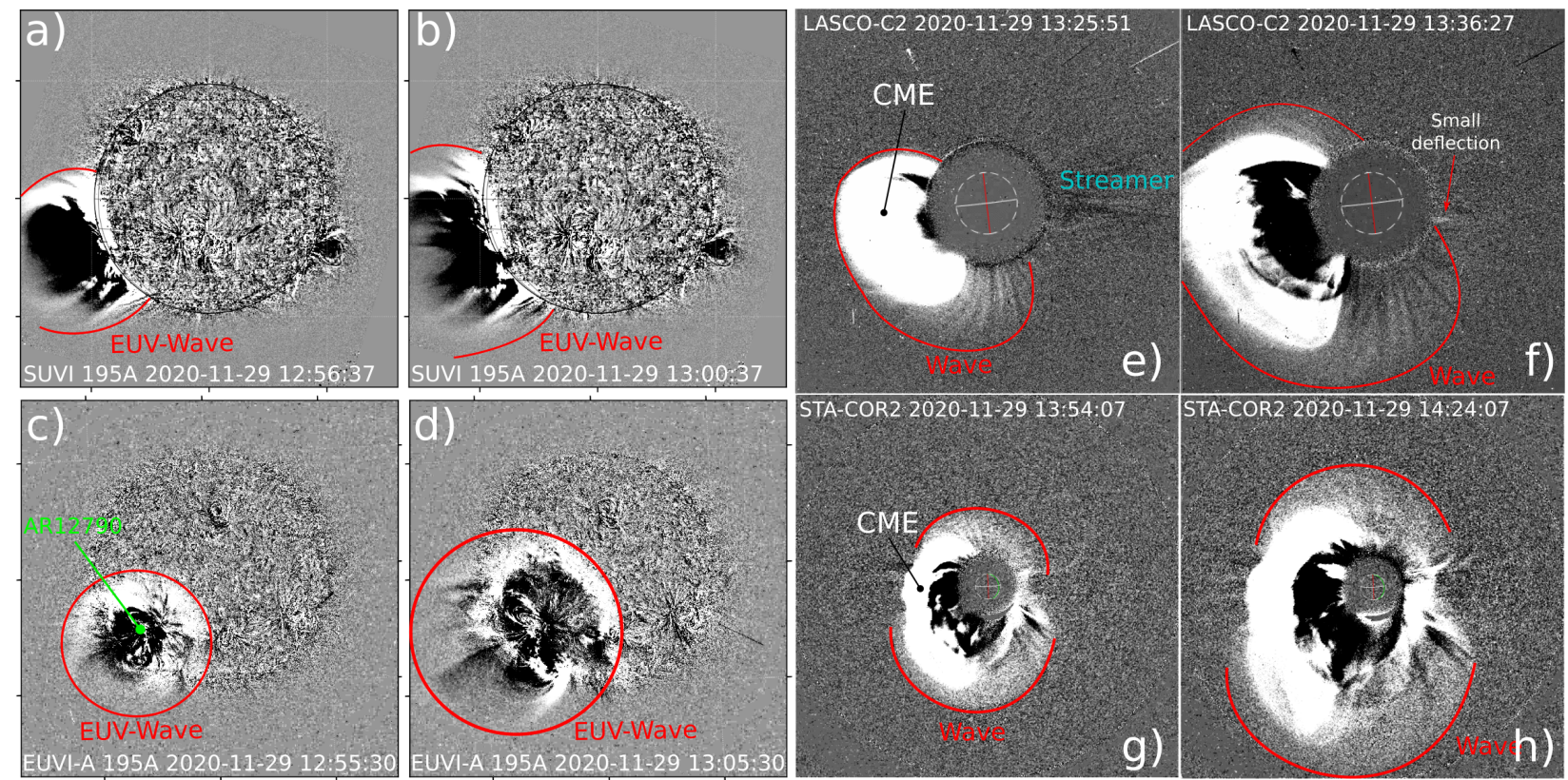

Fig. 3. Remote sensing observations of the EUV wave, the CME, and the white-light shock wave from two different viewpoints. Panels $a-d$ : EUV running-difference images recorded nearly simultaneously from SUVI ( $a$ and $b$ ) and STEREO-A EUVI $(c$ and $d$ ) at $195 \AA$ during the early stages of the EUV wave expansion in the low corona. The EUV wave front is labeled and outlined with the red lines. Panels $e-h$ : running-difference images in white-light from SOHO/LASCO-C2 $(e$ and $f$ ) and STEREO-A/COR2 ( $g$ and $h$ ). The CME is labeled and the white-light shock wave is labeled and outlined with red lines.

count rate rise fairly quickly after the type III radio burst associated with the M4.4 solar flare at $\sim 13: 11$ UT on November 29, while the $0.5 \mathrm{MeV}$ protons and $150 \mathrm{keV}$ electrons show a more gradual increase. The region around the shock (which arrives at PSP on November 30, 18:35 UT) is sufficiently turbulent that the $0.5 \mathrm{MeV}$ protons are well confined and strongly peak at the time of the shock passage. Surprisingly, the peak in the electron count rates (for the two energy channels shown in Fig. 1) is coincident with the shock arrival. It is unlikely that these are locally accelerated by the shock; possibly, they are a trapped population due to the narrowing region between the two ICMEs. After the passage of the shock and sheath region, the SEP intensity drops dramatically as PSP enters the ICME, as is often seen in magnetic clouds (see details in Cohen et al. 2021).

\subsection{Remote sensing observations}

Previous studies (see, e.g., Park et al. 2013) have proposed that widespread SEP events are associated with EUV waves propagating far from the parent active region, or by extended CMEdriven shocks, which inject particles onto field lines that are poorly connected to the active region. For this event, EUV observations of the low corona show that an EUV wave was launched in connection with the solar flare from NOAA AR 12790. The EUV wave appears as a circular wave expanding away from the $\mathrm{AR}$ and lasting for more than one hour. During this time it covers a large distance over the visible disk as seen from STEREOA. Figure 3 shows running-difference images of SUVI (panels a and b) and STEREO-A EUVI (panels c and d) at $195 \AA$ taken at an early stage of the EUV wave expansion with the front of the EUV wave indicated in the images. The EUV observations show that the wave forms at $\sim 12: 47 \mathrm{UT}$, two minutes after the hard X-ray onset time from SOHO/EPHIN (see Fig. 4). We measured that the EUV wave propagates on the solar disk with an average speed of $500 \mathrm{~km} \mathrm{~s}^{-1}$. North and northwest of the AR as seen from
STEREO-A, the wave seems to be brighter and stronger and can be clearly traced until $\sim 13: 30$ UT in SUVI images, when located near central meridian as viewed from Earth. After this time, it is difficult to trace the propagation of the wave on the visible disk.

White-light coronagraphic observations recorded a fast and relatively wide CME together with a shock wave observed as a bright front around the CME. Figure 3 shows running-difference images from SOHO/LASCO-C2 (panels e and f) and STEREO$\mathrm{A} / \mathrm{COR} 2$ (panels $\mathrm{g}$ and $\mathrm{h}$ ) with the front of the shock wave indicated and CME labeled. The CME emerges above the eastern limb in the SOHO/LASCO-C2 images at $\sim 13: 26$ UT. From the viewpoint of STEREO-A/COR2, it is observed as a Halo-CME. The CME first appears in the COR2 images at $\sim 13: 24$ UT. The LASCO-C2 image in panel $\mathrm{f}$ also shows a small streamer deflection at the west limb, suggesting that the pressure wave associated with the CME probably reached very distant locations relative to the flare higher in the corona. From a 3D reconstruction of the CME and the white-light shock wave we estimate that at the radial direction the CME propagates at an average speed of $\sim 1500 \mathrm{~km} \mathrm{~s}^{-1}$ while the shock wave propagates faster with a speed of around $1800 \mathrm{~km} \mathrm{~s}^{-1}$. Both the CME and the white-light shock wave seem to decelerate above $\sim 10 R_{\odot}$. A detailed analysis of the CME and the associated shock wave kinematics will be presented in a subsequent study.

\subsection{Radio and X-ray observations}

Solar radio and X-ray emissions provide insight into particle acceleration at and near the Sun in this event. X-ray light curves and dynamic radio spectrograms are displayed in Fig. 4. The top panel shows GOES observations in the nominal wavelength band $0.1-0.8 \mathrm{~nm}$ (photon energies $2-12 \mathrm{keV}$ ). The time derivative of the soft X-ray emission during its rise phase, plotted by the red curve, is a well-known proxy of the hard X-ray emission from nonthermal electrons and was recently proposed to be a proxy of 

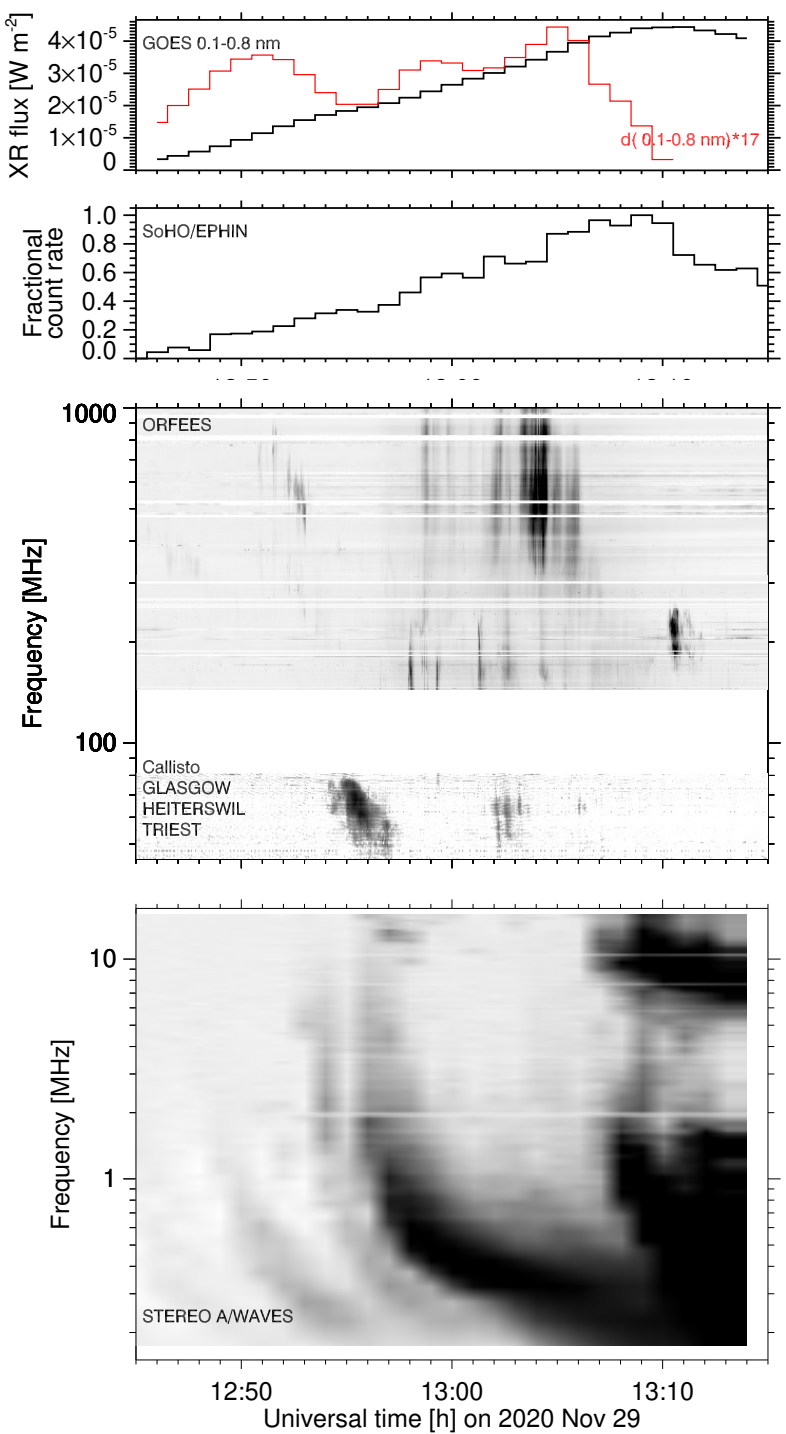

Fig. 4. Time history of the X-ray emission and dynamic spectrograms of the 2020 November 29 solar burst. From top to bottom: (1) time history in soft X-rays and its time derivative (red); (2) count rate time history due to hard X-rays in the SOHO/EPHIN detector; dynamic radio spectrograms in the frequency ranges (3) $144-1000 \mathrm{MHz}$, (4) $41-85 \mathrm{MHz}$, (5) $0.15-16 \mathrm{MHz}$.

the injection function of solar energetic electrons into the heliosphere (Steyn et al. 2020). Its time variation indicates different episodes of electron acceleration during the flare. The second panel from top shows the response to somewhat harder X-rays in the count rate of detector-A from SOHO/EPHIN, which is sensitive to photons with energies in the order of tens of $\mathrm{keV}$ (Kühl et al. 2020). As for the soft X-ray light curve, the rise time profile at EPHIN shows little structure, probably due to the fact that the footpoints of the hard X-ray source, which usually show time fluctuations, are occulted to observers on the Sun-Earth line in this event.

Dynamic spectra of the radio emission from the low corona $(1000 \mathrm{MHz})$ to $0.13 \mathrm{AU}(0.15 \mathrm{MHz})$ are displayed in the three bottom panels of Fig. 4. Bursts at dm-m-wavelengths $(1000-45 \mathrm{MHz})$ accompany the hard X-ray emission. Three time periods can be distinguished:

Firstly, the emission between 12:51 and 12:58 UT consists of an initial faint feature between 950 and $350 \mathrm{MHz}$ with evidence

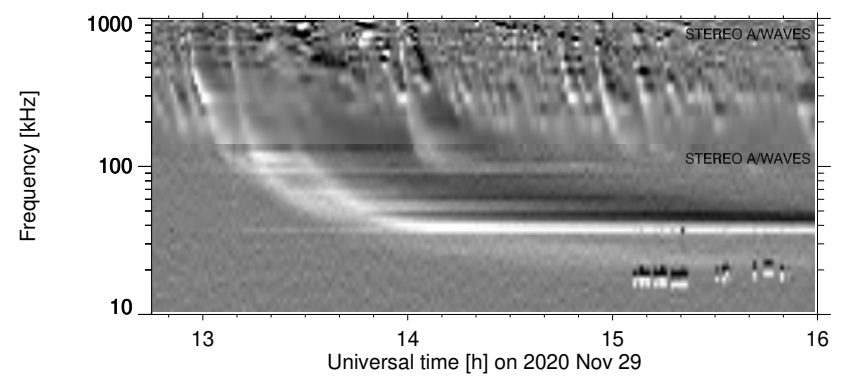

Fig. 5. Frequency-difference spectrum observed by STEREO-A in the range $(0.01-1) \mathrm{MHz}$ (the plotted range is $\pm 7.5 \%)$ in the 2020 November 29 solar event.

of two bands (central panel). The bands start to drift toward lower frequencies at 12:51:30 UT and fade near 12:53 UT. In the $81-45 \mathrm{MHz}$ band (second from bottom) a type II burst is observed between 12:54 and 12:57 UT. The emission between 350 and $950 \mathrm{MHz}$ is probably an early manifestation of the disturbance that later shows up as the type II burst, that is similar to a precursor as identified by Klassen et al. (1999). The type II burst continues down to $10 \mathrm{MHz}$ and ends there near 12:58 UT. On its low-frequency side it is accompanied by type III bursts observed by STEREO-A (bottom panel).

Secondly, after the type II precursor the radio spectrum consists of a series of short broadband bursts between 12:57 and 13:08 UT. They start above $1000 \mathrm{MHz}$ together with a new rise in the soft X-ray derivative and extend down to $45 \mathrm{MHz}$, possibly to $14 \mathrm{MHz}$. They have some spectral structure with distinct emissions above and below $300 \mathrm{MHz}$ during the first minutes. These bursts differ from type III bursts in that they have a welldefined low-frequency cutoff and show no evidence of a drift toward lower frequencies or of a temporal broadening at low frequencies. They are broadband pulsations, that is, a typical fine structure of type IV bursts (see Aurass et al. 2003, and references therein).

Lastly, no later emission is detected by the spectrographs in the $1000-45 \mathrm{MHz}$ band. At frequencies below $16 \mathrm{MHz}$, that is, at decameter-to-hectometer (DH) wavelengths, strong emission starts at 13:06 UT with a well-defined low-frequency edge near $5 \mathrm{MHz}$. It continues until 13:40, with a drift of the low-frequency edge toward lower frequencies, and is followed until 14 UT by a patch of radio emission at similar wavelengths but without a systematic drift. PSP observed the same spectral feature until 13:40. The drifting burst between 13:06 and 13:40 UT is a type II burst. On its low-frequency side it is accompanied by type III bursts. While they overlap in time with the initial phase of the type II burst, they start at higher frequencies above the $16 \mathrm{MHz}$ limit of the STEREO/WAVES receiver.

A dynamic spectrogram at frequencies below $1 \mathrm{MHz}$ is displayed in Fig. 5. It shows the relative difference between flux densities in adjacent frequencies (i.e., the difference divided by the average). White shading marks regions where the flux density increases with increasing frequency. At frequencies above $150 \mathrm{kHz}$ the type III bursts related to the eruptive events are mixed with an unrelated type III storm, while at lower frequencies only type III bursts from the eruptive event are seen. Continuous type III bursts through the band are seen near $1 \mathrm{MHz}$ during the dm-m-wave type II burst (12:50-12:58) and at the start of the DH type II burst (13:08) and at lower frequencies $(300 \mathrm{kHz})$ near 13:20 UT. These type III bursts behave differently at low frequencies: the one accompanying the early meterwave type II burst merges near $100 \mathrm{kHz}$ with the later one that 
has a steeper leading edge in the dynamic spectrogram. The lowfrequency edge of the second type III burst continues down to about $20 \mathrm{kHz}$, as shown by the white halo on the low-frequency side of the type III spectrum. As it reaches $20 \mathrm{kHz}$ near 15:05 UT, several intense packets of Langmuir waves are observed until 15:50 UT. They demonstrate that the spacecraft intercepts at least some of the electron beams producing the type III burst. STEREO-A is hence connected to the source region of these electron beams, launched in the corona during the DH type II burst. The travel time to 1 AU between 13:06 and 15:05 UT corresponds to an average exciter speed of $8.4 \times 10^{-2} c$ traveling 1.2 AU, which is slow (energy $2 \mathrm{keV}$ ), but still consistent with the energy range usually quoted for the electron beams generating Langmuir waves at $1 \mathrm{AU}$ (Ergun et al. 1998). The onset of the Langmuir waves is consistent with the first arrival of electrons near $5 \mathrm{keV}$ at STEREO-A (Fig. 6). The low-frequency behavior of the previous type III burst, together with the fact that Wind/WAVES does not detect this type III burst, suggests that the electron beams accelerated at the meter-wave type II shock traveled along a different path, which was not intercepted by STEREO-A. While STEREO-A detected Langmuir waves, demonstrating a magnetic connection to the source region of the electrons despite the large longitudinal distance of the nominal Parker spiral from the flare site (see Table 1), none were seen at Wind, PSP (Bale, priv. comm.) or SolO (Maksimovic, priv. comm.).

\section{Analysis of SEP onsets}

\subsection{Timing of energetic particles and velocity dispersion analysis}

In order to estimate the solar release time of the energetic particles we have performed the so-called time shift analysis (TSA) and the velocity dispersion analysis (VDA). Both methods are commonly used to identify the release time of energetic particles at the Sun (see, e.g., Krucker et al. 1999; Gómez-Herrero et al. 2021) and multiple studies (e.g., Lintunen \& Vainio 2004; Saiz et al. 2005; Vainio et al. 2013; Laitinen et al. 2015) have discussed the uncertainties and limitations of these methods. We note that the VDA uncertainties assumed here are valid only under the assumption that there is negligible cross-field diffusion.

The VDA is based on the assumptions that particles with different energies are injected at the same time and that the first arriving particles travel nearly scatter-free along the same path to the observer. From the arrival times of particles with different energies both the effective path length and the common solar injection time can be derived. The two parameters are often obtained by a linear fit of the onset times plotted vs. particle velocities given in $c / v$. The TSA assumes that the particles propagate scatter-free along a given path length. Individual solar release times for particles with different velocities are then obtained by time shifting the arrival time to $t_{\text {rel. }}=t_{\text {arr. }}-L / v$.

Both methods require accurate arrival times of the first particles reaching the observer. These times are usually identified from intensity-time-profiles using different methods such as the so-called $3 \sigma$-method (see, e.g., Krucker et al. 1999), the Poisson-CUSUM method (see, e.g., Huttunen-Heikinmaa et al. 2005; Xu et al. 2020) or by simply identifying the onset by eye. Onset times determined with these methods are usually delayed compared to the actual arrival of the first particles since the measured signal has to rise above a certain background first before it becomes detectable. The delay of the onset times can
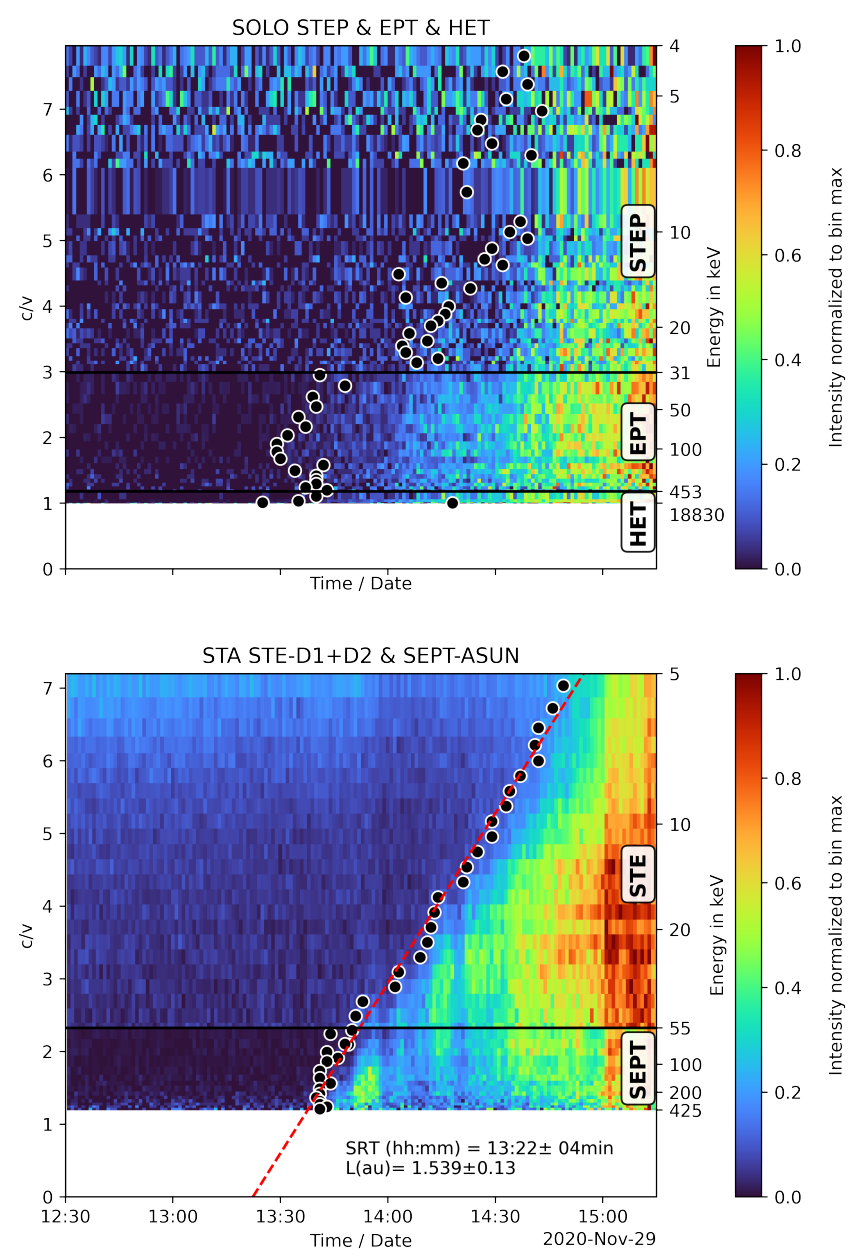

Fig. 6. 60 second time resolution $c / v$ vs. time plots for electrons observed by SolO STEP, EPT, and HET (top panel) and STEREO STE D1 + D2 and SEPT-ASUN (bottom panel). The color scale represents intensities normalized to maximum intensity in the respective energy channel. Black horizontal lines separate the instruments. Black Points mark the onset times derived with the Possion-CUSUM method. The red dashed line in the bottom panel represents the linear fit for VDA.

be made worse by low statistics, gradual increases, or high pre-event backgrounds from either instrumental effects or from pre-occuring events. Additionally, instruments with limited fields-of-view (FOV), not covering a full $4 \pi$ solid angle, may not observe the actual arrival of the first particles if the magnetic field vector and the resulting particle trajectories are outside of the FOV.

It is important to note that while the above effects cause delays to the observed onsets, assessing their influence on the determination of the particle solar release times is not as straightforward. The delays on observed onsets are typically different for different energies, which may cause the VDA-derived solar release times to be early as well as delayed with respect to the real release time (Laitinen et al. 2015).

In general, the results of VDA should be interpreted with caution. The fundamental assumptions of a simultaneous injection of particles with different energies as well as their scatterfree transport may not apply for specific events.

Scattering in the interplanetary medium can broaden the intensity time profiles observed far from the source and the following slower rise of the observed particle intensities tends to cause significant delayed onset detection. Thus, if strong 
scattering is involved in the particle transport, the solar injection times, and path lengths determined by VDA can have significant errors (Laitinen et al. 2015).

To overcome uncertainties in the onset determination Zhao et al. (2019) proposed an extension to the VDA where they considered the times when the flux reached certain ratios of the peak flux instead of the onset times. While this extension would allow us to avoid uncertainties in the onset determination, its application is impracticable for the here discussed event. Given the long rising phase of particle intensities and the modulation of the time profiles by transient structures the unambiguous determination of the relevant peak fluxes in different energy channels is not possible for the here discussed event.

In this work, we apply the VDA only for measurements that show a prompt and clear increase above the background intensities during the early rise phase. Yet, a detailed deconvolution and quantification of the potential sources of errors in the VDA is not feasible with the data presented here. Further studies including simulations of the SEP transport have to confirm the solar release and injection times derived for this event by VDA.

Here we use the Poisson-CUSUM and the $3 \sigma$-Method to derive onset times. We applied the Poisson-CUSUM method to data sets with low statistics where the $3 \sigma$-Method would require extensive down-sampling of the native time or energy resolution. The $3 \sigma$-Method was applied to time profiles with higher statistics.

Considering first VDA, Fig. 6 shows the $c / v$ vs. time plots for electron measurements from STEP, EPT, and HET on SolO (upper panel) as well as STE and SEPT on STEREO-A (lower panel). The colorbar shows the intensity normalized to the maximum intensity in each energy channel. The black horizontal lines separate the individual instruments and the black points mark the onset times of each energy channel derived with the PoissonCUSUM method.

For STEREO-A we combine measurements from the SEPTASUN telescope, that is pointing away from the Sun, together with measurements from the D1 and D2 detectors of Suprathermal Electron Telescope (STE). These apertures were chosen because they share a common FOV and were aligned close to the local magnetic field direction during this time period. Therefore, they likely observed the earliest electrons arriving at the spacecraft. A further description of the multi-sector measurements is given in Sect. 5.2.

VDA results using the onset times determined for SEPT and STE are included in the lower panel of Fig. 6. The red dashed line represents a linear fit through the onset times for the various energy channels starting at $5 \mathrm{keV}$. The fit yields a solar release time of 13:22 UT \pm 4 min as well as an effective path length of $(1.54 \pm 0.13)$ AU. $3 \sigma$ fitting errors were determined by bootstrapping the fit with random samples of half the data points.

While the onset times derived from STEREO-A electron observations follow a clear dispersion and are in general agreement with the linear fit shown, onset times derived from SolO electron observations in the top panel of Fig. 7 are more ambiguous. The flux increase observed by SolO/STEP, SolO/EPT, and $\mathrm{SolO} / \mathrm{HET}$ is not as prompt and well defined as the equivalent STEREO-A observations. The smaller geometry factors of SolO/EPT and SolO/STEP compared to those of STEREO/SEPT and STEREO/STE generally lead to worse statistics while observing similar differential fluxes. The onset times derived from SolO/STEP are inconsistent with the SolO/EPT onsets and likely too late. This discrepancy is probably related to the slightly gradual increase of the event and the different signal-tobackground ratios of the two instruments. Also, the onset times
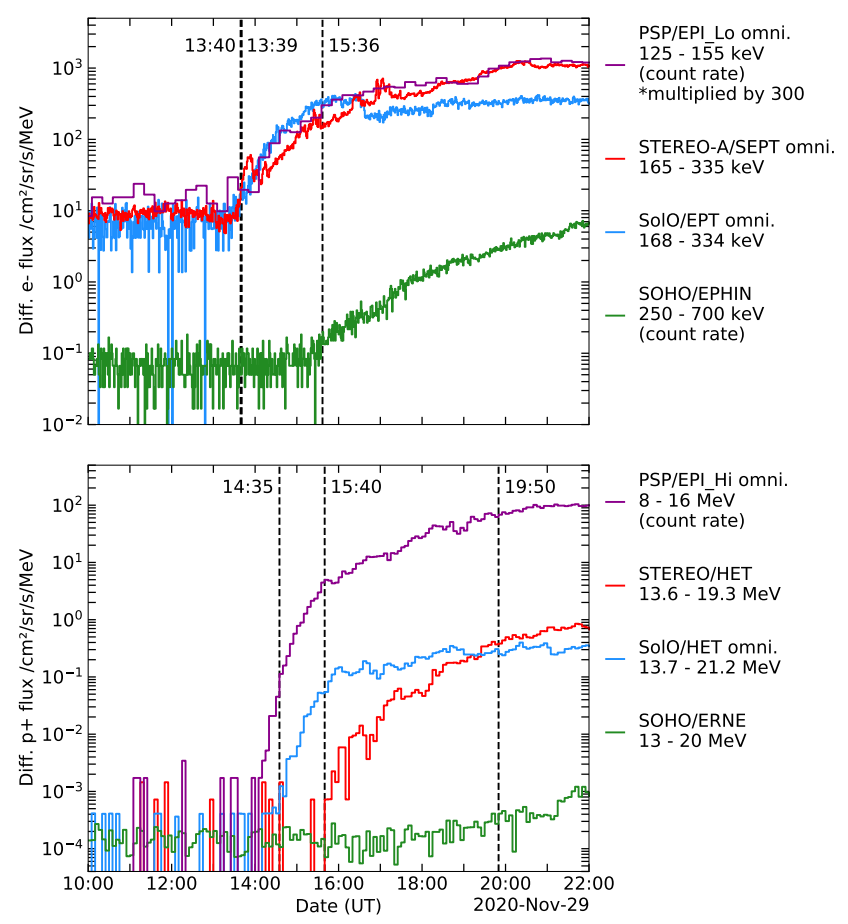

Fig. 7. Electron and proton fluxes observed during the onset phase. Top panel: 1-min averaged electron fluxes observed by SolO/EPT, STEREO-A/SEPT, and SOHO/EPHIN together with 15-min averaged electron count rates measured by PSP/EPI-Lo. Bottom panel: 10min averaged proton fluxes observed by SolO/HET, STEREO/HET, SOHO/ERNE and proton count rates measured by PSP/EPI-Hi. $3 \sigma$ onset times are marked by dashed lines.

of $>200 \mathrm{keV}$ electrons observed by SolO/EPT are seemingly too late compared to onset times at lower energies. This is likely related to the worse signal-to-background ratio present at higher energies. The onset times derived from SolO/HET observations suffer also from generally low statistics.

The large uncertainties of the onset times determined from SolO observations prevented us from performing a VDA on these data sets. Instead we used the earliest observed onset of relativistic electrons at 13:25 UT seen by HET at $2.4-6.0 \mathrm{MeV}$ and a path length of 0.94 AU determined from ENLIL (Table 1) to estimate a solar release time of 13:17 UT using TSA. Similar restrictions also prevented us from performing VDA for nearEarth observations, where the actual onset times are difficult to determine from the slow, gradual flux increase.

Figure 7 shows omni-directional electron (top panel) and proton (bottom panel) intensity-time profiles at the different spacecraft during the event onset. The omni-directional profiles are obtained by combining multiple energy channels and measurements from the different telescopes of each instrument. Onset times identified by the $3 \sigma$-Method are shown in dashed lines. The onset times of near-relativistic electrons $(125-335 \mathrm{keV})$ observed by SolO at 13:40 $\pm 11 \mathrm{~min}$ and STEREO-A at 13:39 $\pm 1 \mathrm{~min}$ are almost identical. The onset of $250-700 \mathrm{keV}$ electrons observed by SOHO at 15:36 $\pm 19 \mathrm{~min}$ is delayed by about two hours compared to the onsets at STEREOA and SolO.

The onset times determined from $\sim 13$ to $21 \mathrm{MeV}$ protons measurements by SolO, STEREO-A, and SOHO are 14:35 $\pm 5 \mathrm{~min}(\mathrm{SolO}), 15: 40 \pm 11 \mathrm{~min}($ STEREO-A) and 19:50 \pm 5 min (SOHO). The given uncertainties are estimated by the difference of $3 \sigma$ and $5 \sigma$ onset times. If both of these onset times 
Table 2. Timeline of the 2020 November 29 SEP event.

\begin{tabular}{|c|c|c|c|c|c|c|}
\hline Date & $\begin{array}{l}\text { Estimated solar release } \\
\text { time (UT) }\end{array}$ & $\begin{array}{l}\text { Delay w.r.t. } \\
\text { Type III(1) (min) }\end{array}$ & $\begin{array}{l}\text { Observed onset } \\
\text { time (UT) }\end{array}$ & Observation & Instrument & Mission \\
\hline $29 / 11$ & $12: 26$ & -19 & $12: 34$ & M4.4 X-ray flare onset & GOES & GOES \\
\hline $29 / 11$ & $12: 37$ & -8 & $12: 45$ & Hard X-ray onset & EPHIN & $\mathrm{SOHO}$ \\
\hline $29 / 11$ & $12: 43$ & -1 & $12: 51$ & dm-m- $\lambda$ type II & ORFEES & Earth \\
\hline $29 / 11$ & $12: 45$ & 0 & $12: 53$ & DH type III(1) & WAVES & STA \\
\hline $29 / 11$ & $12: 58$ & +13 & $13: 06$ & DH type II & WAVES & STA \\
\hline $29 / 11$ & $12: 58$ & +13 & $13: 06$ & DH type III(2) & WAVES & STA \\
\hline $29 / 11$ & 13:03 & +18 & $13: 11$ & M4.4 X-ray flare max & GOES & GOES \\
\hline $29 / 11$ & $13: 17$ & +32 & $13: 25$ & CME & $\mathrm{COR} 2$ & STA \\
\hline $29 / 11$ & $13: 19 \pm 5$ min TSA & +34 & $13: 28 \pm 5 \min ^{(c)}$ & $>0.5 \mathrm{MeV} e$ - onset & EPI-Hi & PSP \\
\hline $29 / 11$ & $13: 22 \pm 4 \mathrm{~min} \mathrm{VDA}$ & +37 & $13: 39 \pm 1 \mathrm{~min}$ & $165-335 \mathrm{keV} e$ - onset & SEPT & STA \\
\hline $29 / 11$ & $13: 17 \pm 11 \min ^{\mathrm{TSA}^{(a)(b)}}$ & +32 & $13: 40 \pm 11 \mathrm{~min}$ & $168-334 \mathrm{keV} e$-onset & EPT & SolO \\
\hline $29 / 11$ & $15: 24 \pm 19 \min ^{T_{S A}}{ }^{(a)}$ & +159 & $15: 36 \pm 19 \mathrm{~min}$ & $250-700 \mathrm{keV} e$ - onset & EPHIN & $\mathrm{SOHO}$ \\
\hline $29 / 11$ & $13: 15 \mathrm{VDA}^{(c)}$ & +30 & $14: 30 \pm 5 \min ^{(c)}$ & $8.0-16.0 \mathrm{MeV} p$ onset & EPI-Hi & PSP \\
\hline $29 / 11$ & $13: 53 \pm 5 \operatorname{min~TSA}^{(a)}$ & +68 & $14: 35 \pm 5 \mathrm{~min}$ & $13.7-21.2 \mathrm{MeV} p$ onset & HET & SolO \\
\hline $29 / 11$ & $14: 47 \pm 10 \min \mathrm{TSA}^{(a)}$ & +122 & $15: 40 \pm 10 \mathrm{~min}$ & $13.6-19.3 \mathrm{MeV} p$ onset & HET & STA \\
\hline $29 / 11$ & $18: 54 \pm 5$ min $\operatorname{TSA}^{(a)}$ & +369 & $19: 50 \pm 5 \mathrm{~min}$ & $13.0-20.0 \mathrm{MeV} p$ onset & ERNE & $\mathrm{SOHO}$ \\
\hline
\end{tabular}

Notes. ${ }^{(a)}$ Assuming the path length from ENLIL simulations listed in Table 1. ${ }^{(b)}$ The onset of $165-334 \mathrm{keV}$ electrons observed by EPT is likely too late due to a low signal-to-background ratio. Here we are using the earliest onset time of relativistic electrons $(2.4-6.0 \mathrm{MeV})$ observed by HET at 13:25 UT for the TSA. ${ }^{(c)}$ Values for PSP were taken from Cohen et al. (2021).

are identical, the uncertainty is determined by the time resolution of the data used.

The proton onset determined from STEREO/HET could also include a delay because the single STEREO/HET telescope was not oriented along IMF at this time and may not have detected the first arriving particles.

The corresponding injection times of these particles obtained by TSA, while assuming a path length of 0.94 AU for PSP, $0.94 \mathrm{AU}$ for SolO, 1.16 AU for STEREO-A, and 1.24 AU for near-Earth missions, are given in Table 2. We note that the combination of energy channels introduces additional uncertainties in the determination of the particle velocities. Here we use the velocity corresponding to the geometrical mean of these particle energy ranges.

Further we note that the path lengths used here are a simple estimate for the actual path traveled by the particles. As studied by Chhiber et al. (2021) and Laitinen \& Dalla (2019) the turbulent meandering of field lines can cause a significant lengthening of the IMF path lengths. Conversely, Laitinen \& Dalla (2019) found that, in certain constellations, a meandering field line could also establish a magnetic connection that is shorter than the nominal Parker spiral length. Other factors such as the gyration of particles that propagate with an effective pitch angle of $\cos (\theta) \neq \pm 1$ will additionally extend the actual path length (see, e.g., Chhiber et al. 2021).

Obviously, the potential discrepancies between the path lengths assumed here and the actual lengths would directly translate to uncertainties in the solar release times determined by TSA. As the determination of an accurate path length is difficult, we restrict our analysis to the path length determined from ENLIL simulations. Additional Monte Carlo simulations of the magnetic field lines and of particle trajectories, considering the solar wind turbulence, might yield better approximations for the actual path length. These analyses however are beyond the scope of this work and will be the subject of future studies.

The release times of SEPs observed by PSP are studied in detail by Cohen et al. (2021). Unfortunately, the EPI-Hi ion intensity vs. energy data are only available on an hourly cadence during this event. Such coarse time resolution makes onset anal- ysis impractical for calculating particle solar release times. There is, however, a sample of individual ion measurements recorded by EPI-Hi with 1-min time resolution that can be used in inverse velocity analysis. For this, the inverse velocity of each ion was calculated and plotted vs. the particle observation time. A clear edge to the distribution was then fitted by eye to obtain a path length and a solar release time at 13:15 \pm 4 min UT (see Cohen et al. 2021, for details).

Since the determination of the electron EPI instrument response has not been completed, similar analysis of the electron events is not possible. There are electron count rates at 1-min time resolution available from PSP/EPI-Hi/HET, but only categorized by which detector the particle stopped in. This is difficult to convert to an energy range due to the significant variation in incident angles as well as due to scattering in the instrument. However, given that HET's response is primarily to electrons $>0.5 \mathrm{MeV}$, the velocity dispersion of these electrons is fairly small. By examining the onset of these 1-min rates, we estimate the electron onset time to be 13:28 \pm 5 min UT.

\subsection{Anisotropies}

The two top panels of Fig. 8 show the pitch angle distribution (PAD) of near-relativistic (left) and relativistic (right) electrons observed by SolO/EPT and HET, respectively. We note that the SolO/HET PAD is a data product that is not available from any other present heliospheric spacecraft. Corresponding to the energy range shown in the top left panel, the bottom panels show the PADs observed by STEREO A/SEPT (left) and Wind/3DP (right). The first order anisotropy is presented in the bottom panels of Fig. 8. In case of the four-sector telescopes, the anisotropies were determined using the summation method described by Brüdern et al. (2018). For Wind's eight-sector measurements an integration of the fitted PAD was used (e.g., Dresing et al. 2014). It shows that Wind observes only a very weak anisotropy due to anti-sunward propagating electrons with an average value of $\langle A\rangle=0.3$ for the first hour of the event. However, this weak anisotropy lasts from the time of the onset around 16 UT on November 29 until 

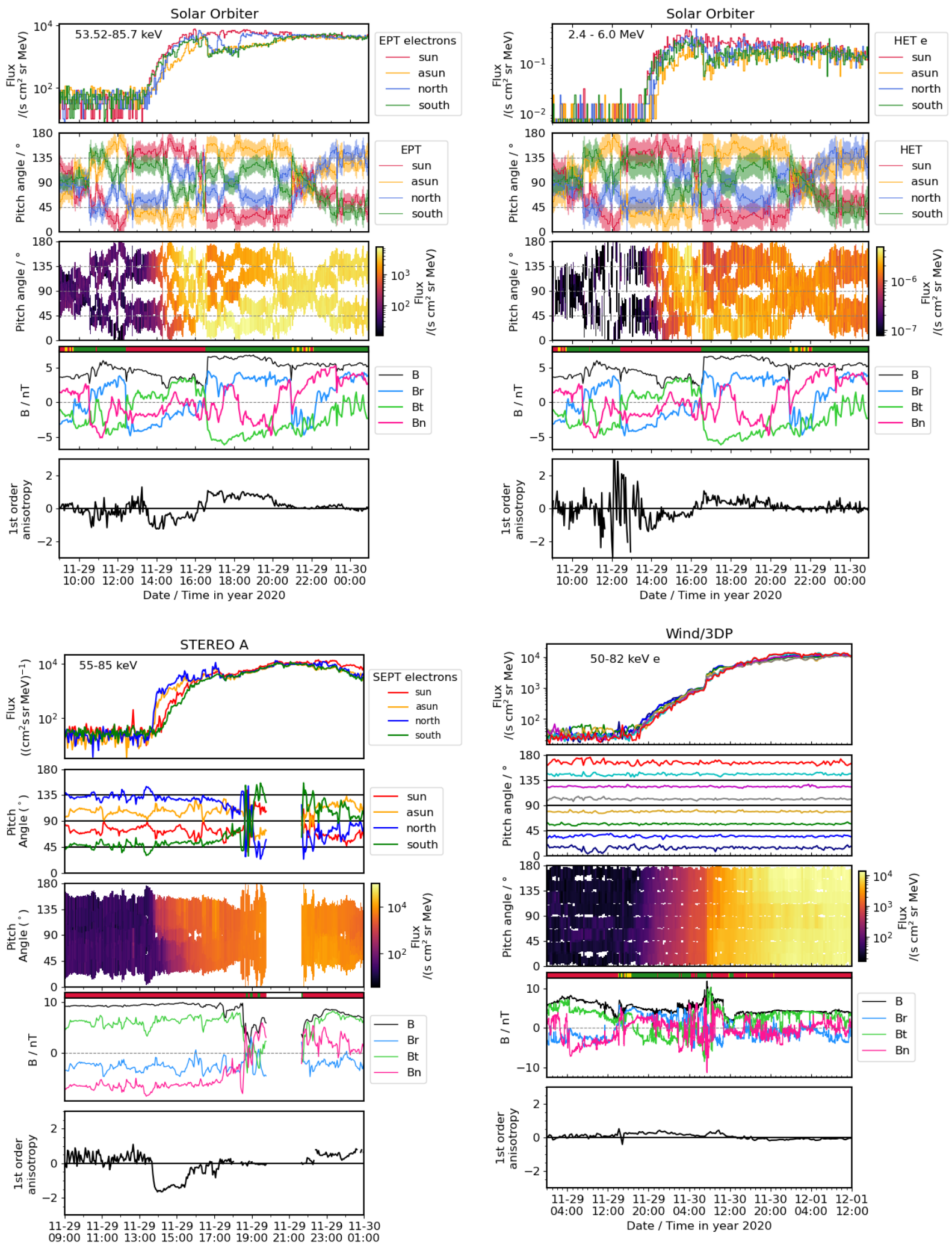

Fig. 8. Pitch angle distributions of energetic electrons observed by SolO/EPT (top left), SolO/HET (top right), STEREO A/SEPT (bottom left), and Wind/3DP (bottom right). Top panels: sectored electron fluxs of $\sim 50-80 \mathrm{keV}$ (bottom figures and top left figure) and $2.4-6.0 \mathrm{MeV}$ (top right, HET) observed in the four viewing directions of the telescopes (in case of STEREO and SolO) or binned into eight sectors for Wind. Second panel: corresponding pitch angles covered by the different viewing directions or sectors, third panel: pitch angle distributions of the electrons (flux in color coding). Fourth panel: magnetic field magnitude and RTN components and bottom panel: first order anisotropy. The colored band on top of the magnetic field panel denotes the in situ magnetic field polarity with red (green) marking negative (positive) polarity and yellow denoting unclear polarity periods. We note the longer time period of the figure showing Wind data. 
almost $10 \mathrm{UT}$ on November 30 . While the anisotropy during the beginning of the event is caused by anti-sunward propagating electrons, the anisotropic period from $\sim 07$ to $\sim 10$ UT on November 30 is caused by sunward propagating electrons as illustrated by the opposite (negative) field polarity during this period. The long-rise time of the event together with the weak anti-sunward anisotropy early in the event suggest a prolonged injection close to the Sun, whereas the short interval with weak sunward anisotropy might result from local effects produced by the CIR identified in the right column of Fig. 1. Presumably, the injection region on the Sun did not extend toward the magnetic footpoint of Wind at the Sun as this would likely result in a larger anisotropy at the spacecraft. A certain amount of perpendicular diffusion might therefore be involved in transporting the particles to the spacecraft. However, detailed transport modeling would be needed to specify the relative contributions of interplanetary transport versus injection size and duration.

SolO observes a stronger anisotropy which is expected due to its much smaller longitudinal separation angle to the parent solar active region as compared with Wind (see Table 1). The absolute value of the first order anisotropy at $\mathrm{MeV}$ energies observed by SolO/HET (Fig. 8, top right, bottom panel) is slightly smaller $(\langle A\rangle=-0.7$ for the first hour) than at near-relativistic energies measured by EPT $(\langle A\rangle=-0.8$, Fig. 8 , top left, bottom panel). This is expected because of the energy dependent diffusion coefficient leading to stronger interplanetary scattering for higher energy electrons (Dröge 2003; Agueda et al. 2014; Strauss et al. 2020). We note that the sign of the first order anisotropy depends on the polarity of the magnetic field and all nonzero anisotropy shown in Fig. 8 is due to anti-sunward propagating electrons except the short period of sunward propagating electrons at Wind as discussed above. Therefore, the switch of negative to positive anisotropy observed by SolO at $\sim 16: 30$ UT is only caused by the change of magnetic field polarity, yielding a duration of more than seven hours which supports the hypothesis of a temporally extended electron injection already suggested by the Wind observations. The largest anisotropy is observed by STEREO/SEPT with a mean value of $\langle A\rangle=-1.4$ during the first hour of the event. The duration of nonzero anisotropy of only about three hours is shorter than at the other spacecraft. However, the vanishing anisotropy could also be due to limited pitch angle coverage, which decreases throughout the event. The large anisotropy observed at STEREO-A is surprising because this spacecraft is far separated $\left(\sim 95^{\circ}\right)$ from the parent active region at the Sun. However, in the light of the detection of local Langmuir waves at STEREO a large anisotropy is expected (see Sect. 4.2). The large longitudinal range covered by STEREO A, SolO, and Wind, showing significant and partially even high electron anisotropies, suggests a spatially extended electron injection or distribution close to the Sun like the class-2 events of Dresing et al. (2014). The observation of anti-sunward directed anisotropies in regions of different field polarity (as indicated by the red and green color bands in Fig. 8) suggest that the injection of particles occurred on both sides of the heliospheric current sheets. There is the possibility that the extent of the distribution of electrons injected close to the Sun was not as large as the longitudinal range spanned by the observers (as perpendicular transport is likely involved in the particle spread), but it is likely far larger than that provided by a flare injection only.

\section{Summary and discussion}

On 2020 November 29, a solar energetic particle event was observed by spacecraft near Earth (SOHO, ACE, and Wind) and by STEREO-A, PSP, and SolO at widely separated locations spanning $\sim 230^{\circ}$ in heliolongitude and $0.81-0.99 \mathrm{AU}$ in heliocentric distance, providing the first opportunity to study a widespread SEP event using such a constellation of four observers. This study summarizes the observations at the different spacecraft and also highlights the capabilities of the new generation of instruments on SolO.

Remote sensing observations show that the SEP event was associated with a partially occulted M4.4 X-ray flare located in NOAA active region 12790 just behind the east limb as observed from Earth (E98). The flare was accompanied by an EUV wave that expanded away from the AR and covered a large portion of the visible solar disk seen by STEREO-A and Earth. Coronagraphic observations show a fast $\left(1500 \mathrm{~km} \mathrm{~s}^{-1}\right)$ and relatively wide $\mathrm{CME}$ originating from the same $\mathrm{AR}$ and a fast CME-driven shock wave. Type II and multiple type III radio emissions are evidence for the propagation of the CME-driven shock wave and the release of electrons into interplanetary space.

In situ particle observations by four widely separated observers give evidence for the wide spread of SEPs. As summarized in Fig. 1, energetic protons with energies extending to $>50 \mathrm{MeV}$ and near-relativistic and relativistic electrons rapidly filled the inner heliosphere following the solar event. The onset times determined for near-relativistic electrons and tens of $\mathrm{MeV}$ protons, as well as the resulting solar release times determined by VDA and TSA, show a dependence on the longitudinal separation between the solar event and the footpoint of the magnetic field connecting to the spacecraft, as has been previously reported for similar widespread events (e.g., Dresing et al. 2012; Lario et al. 2017; Richardson et al. 2014). The differences in the particle onset and release times at the four locations are presumably determined by, and provide constraints on, particle acceleration and transport processes and require further investigation. Here, we briefly consider two examples of how to interpret these onset and release times that indicate the value of combining observations of widespread SEP events from multiple spacecraft.

In our first interpretation, we compare the solar release times of electrons and ions observed by the different spacecraft with the propagation of the EUV wave. Figure 9 presents a timeline of the events in Table 2. It shows the angular distance of the EUV wave from the AR ( $\star$ markers), which is determined "by eye" from SDO/AIA and STEREO-A/EUVI images. The vertical bars show the angular distance of the spacecraft magnetic field footpoints from the AR from Table 1 assuming Parker spiral field lines; the widths of the bars assume an uncertainty of $\pm 5^{\circ}$. The electron (proton) release times for each spacecraft, inferred from TSA or VDA (with the $1 \mathrm{AU}$ light travel time added), are also shown by $\times$ and + markers, respectively.

Comparing the release times of the energetic particles for each spacecraft with the arrival times of the EUV wave at the corresponding field line footpoints, we do not find a clear relationship between these times. The electron release times determined by TSA for PSP (connected around $70^{\circ}$ from the AR) do appear to coincide with the arrival of the EUV wave at the respective field line footpoint. For SolO, the magnetic footpoint is behind the east limb, preventing us from determining a direct connection of the EUV wave extension to the magnetic footpoint. For an EUV wave speed of $500 \mathrm{~km} \mathrm{~s}^{-1}$ the electron release times determined by TSA for SolO (connected around $90^{\circ}$ from the AR) would also coincide with the arrival of the EUV wave to the magnetic footpoint. For STEREO-A it is, again, difficult to trace the EUV wave to the spacecraft footpoint. Moreover, Fig. 9 suggests 


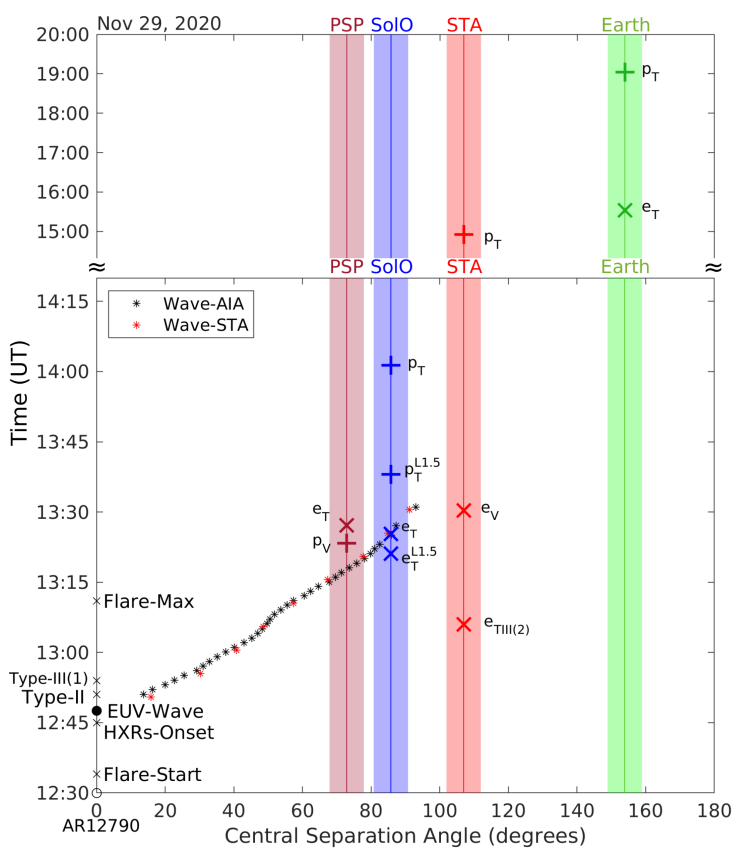

Fig. 9. Comparison of the inferred particle release times with the arrival time of the EUV wave at the spacecraft field line footpoints. The $\star$ markers show the angular distance of the EUV wave from the AR. The release times for electrons and protons are shown by $\times$ and + markers for each spacecraft. The angular distance of the magnetic footpoint from the active region for each spacecraft is shown by the vertical bars, which assume an uncertainty of $\pm 5 \mathrm{deg}$.

that the electron release may have happened before the EUV wave reached the STEREO-A footpoint. Another consideration for STEREO-A is that the second type III radio burst (Sect. 4.3), starting at 13:06 UT, that later reaches the local plasma frequencies at STEREO-A, indicates that some electrons were released onto the field line connecting to STEREO-A prior to the release time determined by VDA (see the marker labeled with $e_{\mathrm{TIII}(2)}$ ). Considering observations near Earth, we find no obvious evidence that indicates that the EUV wave reached the magnetic footpoint. Even if a magnetic connection was established close to the solar surface, the electron release time would be considerably delayed relative to the intersection of the wave with the field line footpoint. For the proton release times, their relation with the arrival of the EUV wave at the footpoints of the different spacecraft seems to be worse. At PSP, the proton release time may be consistent, within errors, with the arrival of the EUV wave at the field line footpoint, whereas at SolO the release times are 15-35 min later. At Earth, even if a connection was established close to the solar surface, the proton release times would be significantly delayed from the extrapolated arrival time of the wave at the field line footpoint.

There are several possible scenarios that could help to account for the above discrepancies. The presence of open magnetic field lines rooted in the active region, that strongly diverge with height, could help to account for the early electron arrival at STEREO-A, since the electrons could be rapidly transported longitudinally in the corona over several tens of degrees (Klein et al. 2008; Klassen et al. 2018). The significant anisotropy of the first arriving electrons and the detection of Langmuir waves at STEREO-A are complementary pieces of evidence that nonnominal magnetic connections are an important element of this widespread event. To account for the delayed proton release times, a possibility is that the properties of the shock and ambient
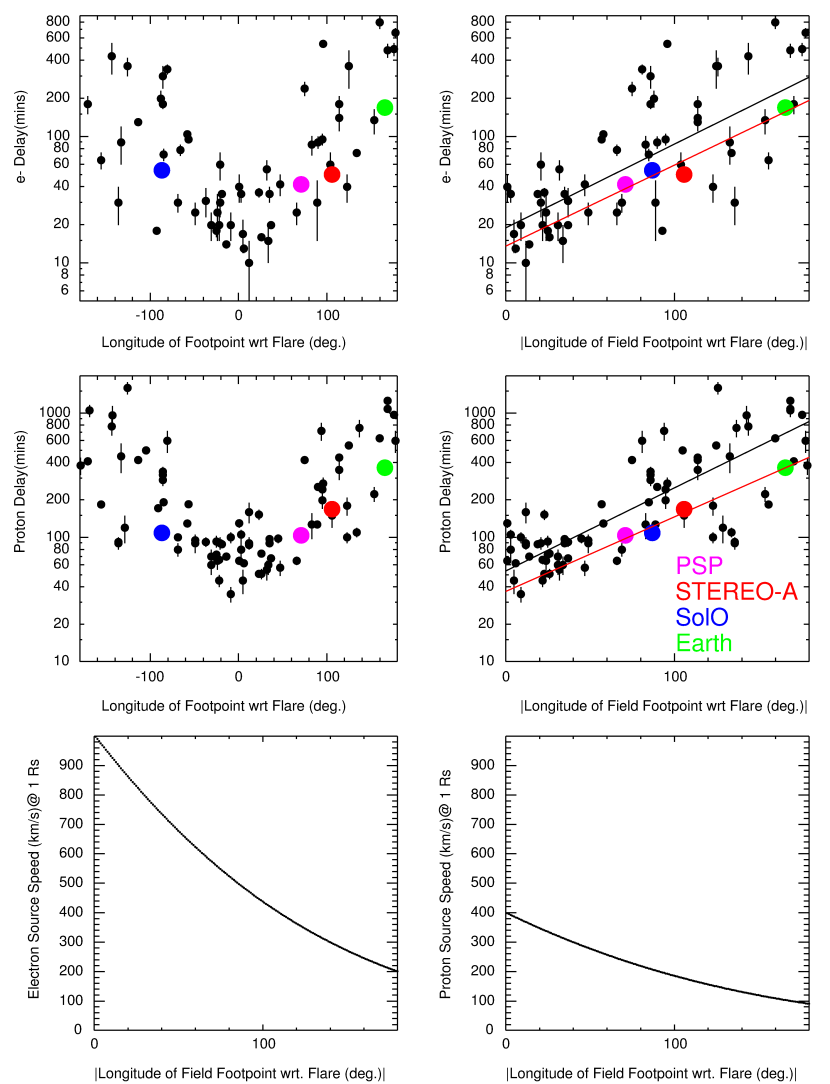

Fig. 10. Comparison of electron (top panels) and proton (middle panels) onset delays vs. connection angle for the November 2020 SEP event (colored circles, indicating the observing spacecraft) with those for $\sim 0.7-4 \mathrm{MeV}$ electrons and $14-24 \mathrm{MeV}$ protons in the cycle $24 \mathrm{SEP}$ events observed at both STEREO spacecraft and near the Earth discussed by Richardson et al. (2014) (black circles). The bottom panels show the "source speeds" from the flare to the field line footpoint as a function of connection angle for electrons (left) and protons inferred from the November 2020 event onset delays.

medium affect the acceleration of electrons and protons differently. Another explanation could be a prolongation of the path length for protons caused by a turbulent random-walk or by the meandering of field lines in the corona and interplanetary space, as modeled by Laitinen \& Dalla (2019). This would increase the proton path lengths from those assumed in TSA and would hence reduce the time differences between the proton release times and the EUV wave connection times. Application of this model considerably reduces the time difference for SolO (see markers labeled with $p_{\mathrm{T}}^{\mathrm{L} 1.5}$ and $e_{\mathrm{T}}^{\mathrm{L} 1.5}$ in Fig. 9 which assume a prolonged path length of 1.5 AU) but not for STEREO-A. In summary, the actual particle acceleration and release processes are likely to be far more complex than assumed in constructing Fig. 9, and further detailed modeling is required to evaluate each of those aspects.

In our second interpretation, we compare the observed electron and proton onset delays in the November 2020 event with observations of similar SEP events during solar cycle 24 , and consider the implications of a simple interpretation of these delays. Richardson et al. (2014) examined the connectionangle dependence of electron and proton delays, derived from observed particle event onset times relative to the onset of the associated type III radio emissions, for a sample of SEP events observed at both STEREOs and near-Earth spacecraft in solar cycle 24 . Figure 10 compares these results with the similar 
delays during the November 2020 event. The top left panel shows (black circles) the near-relativistic electron delays vs. connection angle for the cycle 24 events (where the connection angle is positive if the spacecraft field line footpoint is to the west of the flare), illustrating the general increase in the onset delay with increasing connection angle. The colored circles show the similar results for the November 2020 event, based on information from Table 1. The top right panel shows the same results plotted vs. the absolute value of the connection angle together with the $\log$-linear fits for all the cycle 24 events and for the November 2020 event. We note that the electron delays in the 29 November 2020 event are similarly ordered by increasing connection angle. They are slightly lower than the average for the cycle 24 events. In particular, the electron onset delay for perfect connection (zero connection angle) inferred from the fit (red line) to the observations for the 29 November 2020 event is $14 \pm 4$ min, which is comparable to the intervals between the solar release and event onset times in Table 2 inferred from VDA or TSA. The similar delay for the cycle 24 events (black line showing the corresponding fit) is $19 \pm 4 \mathrm{~min}$.

Similar plots for protons are shown in the middle row of Fig. 10, where the cycle 24 results are for $14-24 \mathrm{MeV}$ protons. Again, the delays for the 29 November 2020 event are within the same range but are also less than the average values based on the log-linear fit. The proton onset delay for perfect connection is $37 \pm 6$ min compared to $54 \pm 5 \mathrm{~min}$ for the cycle 24 events (again obtained from the fit). The significance of these shorter delays is unclear. They may be simply due to event-toevent variations or possibly associated with long-term variations in the particle mean free paths (e.g., Dröge et al. 2016) since the cycle 24 events were observed close to solar maximum, while the 29 November 2020 event is early in cycle 25; this requires further investigation. The locations of PSP and SolO inside $1 \mathrm{AU}$ (Table 1) are also not taken into consideration here, but the differences in particle path lengths are unlikely to be sufficient to account for the differences in these delays.

The bottom panels assume (as in Richardson et al. 2014) a simple scenario in which the onset delay at the spacecraft is the sum of a longitudinally-independent particle travel time from the Sun to the spacecraft, given by the delay at zero connection angle as discussed above, and a delay attributed to the time a particle source moving away from the flare at the solar surface, or, alternatively, a particle diffusion process, takes to reach the footpoint of the field line connected to the spacecraft. The electron and proton source speeds for the 29 November 2020 event in the bottom panels are the average speeds required for the electron or proton source to reach a given connection angle, based on the respective log-linear fits in the middle row of the figure. As in Richardson et al. (2014), these speeds decline with increasing connection angle and are higher for electrons than for protons, reflecting the smaller onset delays for electrons. In this simple scenario, the different source speeds suggest that the electron and proton onset delays cannot be accounted by a connection to a single particle source moving away from the solar event near the Sun. On the other hand, a particle speed dependent diffusion process for electrons and protons could account for the observed onset delays. Despite the simplicity of this analysis, the onset times for all four spacecraft involved (including SolO connected to the opposite side of the flare than the other spacecraft) are so well aligned that any combination of three spacecraft allows the onset time at the fourth to be determined with good accuracy. Moreover, onset delays for all near-ecliptic locations around the Sun can be inferred from the fit. Thus, a new capability to make such onset time projections is demonstrated using these first observations of an SEP onset by four in-ecliptic, near-1-AU spacecraft in widely-separated locations with accurate timing.

Further insight into the particle acceleration and transport is provided by the solar radio emissions accompanying this SEP event. The type II radio emissions give evidence for the propagation of a CME-driven shock wave, and multiple type-III radio bursts for multiple releases of energetic electrons into interplanetary space. Type III bursts at the time of the early meter-wave type II burst (type III(1) in Table 2) emanate from the type II lane and are likely accelerated at the shock. These electrons do not seem to reach STEREO A. The type III bursts after 13:06 UT (type III(2) in Table 2) start at higher frequencies than the simultaneous type II burst at decametric-hectometric wavelengths, suggesting that the corresponding electrons, which are detected at STEREO A, come from some acceleration process behind the shock.

A possibility is that they are released from closed structures of the CME as it interacts and reconnects with ambient open field lines. Similar processes resulting in onset delays and long lasting injections were discussed, for instance, by Klein et al. (2005), Dresing et al. (2018) and Pacheco et al. (2019). As already mentioned, the observation of local Langmuir waves and clear velocity-dispersed electron onsets at STEREO-A indicate that the spacecraft directly intercepted some of the electron beams producing the type III burst. This indicates that the spacecraft was directly connected to the source region of at least one of the electron beams despite its wide separation from the initial flare location.

The in situ observations presented in Sect. 4.1 indicate that the propagation of particles can also be influenced by solar wind structures. SolO, STEREO-A and near-Earth spacecraft show features in the SEP intensity-time profiles that are associated with the passage of local solar wind structures, including sector boundaries, that in most cases are likely to be corotating features. This suggests that the large scale 'background' solar wind structures play a role in determining how the particle intensities vary with time at a particular spacecraft, whether by changing the spacecraft connection to the particle source and/or influencing the transport of particles in the inner heliosphere. This requires further study, for example by modeling the structures present in the ambient solar wind, their relationship to the transient structures associated with prior solar eruptions, and their impact on field line connections and particle transport.

This initial examination of the observations presented in this study suggests that aspects of the 2020 November SEP event are both consistent and inconsistent with two idealized extreme scenarios of particle acceleration and transport in such widespread events. In one scenario, SEPs are accelerated along a broad CME shock front with particle propagation mainly parallel to the magnetic field. The longitudinal breadth of the particle distribution arises from the wide acceleration source, and the observed particle onset delays are due to the source reaching the field lines connected to the individual spacecraft. While there is no direct evidence for this scenario in the particle observations as they are presented in Fig. 9, the actual acceleration process could be more complex than assumed when constructing this figure: the longitudinal spreading of the CME shock would be more rapid higher in the middle corona than close to the surface, explaining why the EUV wave expansion might not accurately indicate when magnetic connection of the coronal shock to the observer is established (Zhu et al. 2018). As shown by Posner et al. (1997) and Miteva et al. (2014), the EUV wave expansion speeds may be too low to account for the onset of electron fluxes. 
Different acceleration efficiencies for electrons and protons at quasi-parallel and quasi-perpendicular shocks could then explain the different delays for the two species if a shock connection has been established. However, the long onset delays observed at L1 do seem incompatible with the time taken for the shock to expand as far as the L1 connected field line. More detailed analysis of the shock wave driven by the CME and its particle acceleration efficiency is clearly required before concluding whether this scenario is applicable to this event.

In another scenario, efficient cross-field transport of particles could be the dominant process, so that a narrow source could lead to broad longitudinal particle spread at $1 \mathrm{AU}$. The different onset times of electrons and protons at all four spacecraft, as well as the different "source speeds" for the two species in Fig. 10, might well be compatible with such a scenario. The low values of the first order anisotropy obtained from near-Earth observations together with the more gradual flux increase observed near Earth suggest that cross-field transport is likely involved in the particle spread. However, the relatively large anisotropies measured at SolO and STEREO-A pose strong constraints on the location where the spreading occurs, placing it close to the Sun rather than in the interplanetary medium. Thus, simulations of coronal and interplanetary particle transport are needed to clarify if this scenario is fully consistent with the observations.

Clearly, these two scenarios represent idealized extremes, and a combination of these scenarios might account for the widespread 2020 November 29 SEP event. The observations and simple analyses presented here cannot rule out a scenario in which an expanding source and perpendicular particle transport are both involved in the particle spread. More detailed studies, using 3D models that assume an expanding particle source and incorporate various transport processes, may lead to a plausible explanation for this event.

In conclusion, this initial study of the 2020 November 29 SEP event demonstrates the value of combining observations from multiple spacecraft in the inner heliosphere, together with remote sensing observations, in helping to understand processes of particle acceleration and transport in widespread SEP events. It also demonstrates some of the capabilities of the new instrumentation on SolO. We anticipate that further opportunities to study SEP events using such observations will arise as solar cycle 25 progresses toward maximum and that in particular, interesting observations from unique viewpoints will be available as PSP approaches closer to the Sun and SolO climbs to higher latitudes.

Acknowledgements. Solar Orbiter is a space mission of international collaboration between ESA and NASA, operated by ESA. The CAU Kiel team acknowledges support from the German Federal Ministry for Economic Affairs and Energy, the German Space Agency (Deutsches Zentrum für Luft- und Raumfahrt e.V., DLR) under grants 50OT0901, 50OT1202, 50OT1702, and 50OT2002. A.K. acknowledges financial support from the ANR COROSHOCK project (ANR-17-CE31-0006-01). D.L. acknowledges support from NASA-HGI grant NNX16AF73G and the NASA Program NNH17ZDA001N-LWS. This study has received funding from the European Union's Horizon 2020 research and innovation programme under grant agreement No. 101004159 (SERPENTINE). The U. Turku team acknowledges funding by the Academy of Finland (Grant No. 336809). The UAH team acknowledges financial support by the Spanish Ministerio de Ciencia, Innovación y Universidades FEDER/MCIU/AEI Projects ESP2017-88436-R and PID2019-104863RB-I00/AEI/10.13039/501100011033. L.R.G. is also supported by the European Space Agency, under the ESA/NPI program, and acknowledges the work performed by Laura Balmaceda and Hebe Cremades in the $3 \mathrm{D}$ reconstruction of the CMEs used in the ENLIL simulation. ENLIL simulation results have been provided by the Community Coordinated Modeling Center at NASA Goddard Space Flight Center (GSFC) through their public Runs on Request system (http://ccmc.gsfc.nasa.gov; run IDs Laura_Rodriguez-Garcia_031321_SH_1). The WSA model was developed by
C. N. Arge, currently at GSFC, and the ENLIL Model was developed by D. Odstrcil, currently at George Mason University. We thank the STEREO: SECCHI, S/WAVES; SOHO: LASCO; Wind/WAVES; and SDO/AIA teams for providing the data used in this study. The STEREO SECCHI data are produced by a consortium of RAL (UK), NRL (USA), LMSAL (USA), GSFC (USA), MPS (Germany), CSL (Belgium), IOTA (France), and IAS (France). SOHO is a mission of international cooperation between ESA and NASA. The SDO/AIA data are provided by the Joint Science Operations Center (JSOC) Science Data Processing (SDP). We thank the radio monitoring service at LESIA (Observatoire de Paris) for providing value-added data that have been used for this study. The ORFEES radio-spectrograph (Observations Radio pour FEDOME et l'Etude des Eruptions Solaires) is the result of a partnership between Paris Observatory and the French Air Force. CALLISTO is a radio spectrograph network organized by the Institute of Astronomy, ETH Zurich and FHNW Windisch, Switzerland. We acknowledge data from the instruments in Glasgow (UK), Heiterswil (Switzerland), and Trieste (Italy). The RPW instrument has been designed and funded by CNES, CNRS, the Paris Observatory, The Swedish National Space Agency, ESA-PRODEX and all the participating institutes. I.G.R. acknowledges support from NASA programs NNH19ZDA001N-HSR and NNH19ZDA001NLWS, and the STEREO mission. IRFU team acknowledges support from the Swedish National Space Agency grant 20/136. A.A. acknowledges the support of the Spanish Ministerio de Ciencia e Innovación (MICINN) under grant PID2019105510GB-C31 and through the 'Center of Excellence María de Maeztu 20202023' award to the ICCUB (CEX2019-000918- M). F.C. acknowledges the financial support by the Spanish MINECO-FPI-2016 predoctoral grant with FSE. E.A. would like to acknowledge the financial support by the Academy of Finland (Postdoctoral Grant No 322455). V.K. acknowledges the support by NASA under grants 18-2HSWO218_2-0010 and 19-HSR-19_2-0143. Solar Orbiter magnetometer operations are funded by the UK Space Agency (grant ST/T001062/1). T.S.H. is supported by STFC grant ST/S000364/1. T.L. and S.D. acknowledge support from the UK Science and Technology Facilities Council (STFC; grant ST/R000425/1).

\section{References}

Agueda, N., Klein, K.-L., Vilmer, N., et al. 2014, A\&A, 570, A5

Arge, C. N., \& Pizzo, V. J. 2000, J. Geophys. Res., 105, 10465

Arge, C. N., Luhmann, J. G., Odstrcil, D., Schrijver, C. J., \& Li, Y. 2004, J. Atmos. Solar-Terrest. Phys., 66, 1295

Aurass, H., Klein, K., Zlotnik, E. Y., \& Zaitsev, V. V. 2003, A\&A, 410, 1001

Battarbee, M., Dalla, S., \& Marsh, M. S. 2018, ApJ, 854, 23

Benz, A. O., Monstein, C., \& Meyer, H. 2005, Sol. Phys., 226, 143

Bougeret, J. L., Goetz, K., Kaiser, M. L., et al. 2008, Space Sci. Rev., 136, 487

Brüdern, M., Dresing, N., Heber, B., et al. 2018, Cent. Eur. Astrophys. Bull., 42, 2

Cane, H. V., Reames, D. V., \& von Rosenvinge, T. T. 1988, J. Geophys. Res., 93, 9555

Chhiber, R., Matthaeus, W. H., Cohen, C. M. S., et al. 2021, A\&A, 650, A26

Cliver, E. W., Kahler, S. W., Neidig, D. F., et al. 1995, Proc. 24th Int. Cosmic Ray Conf., 4, 257

Cohen, C. M. S., Christian, E. R., Cummings, A. C., et al. 2021, A\&A, 656, A29 (SO Cruise Phase SI)

Dalla, S., Marsh, M. S., Kelly, J., \& Laitinen, T. 2013, J. Geophys. Res. (Space Phys.), 118, 5979

Dresing, N., Gómez-Herrero, R., Klassen, A., et al. 2012, Sol. Phys., 281, 281

Dresing, N., Gómez-Herrero, R., Heber, B., et al. 2014, A\&A, 567, A27

Dresing, N., Gómez-Herrero, R., Heber, B., et al. 2018, A\&A, 613, A21

Dröge, W. 2003, ApJ, 589, 1027

Dröge, W., Kartavykh, Y. Y., Klecker, B., \& Kovaltsov, G. A. 2010, ApJ, 709, 912

Dröge, W., Kartavykh, Y. Y., Dresing, N., \& Klassen, A. 2016, ApJ, 826, 134

Ergun, R. E., Larson, D., Lin, R. P., et al. 1998, ApJ, 503, 435

Fox, N. J., Velli, M. C., Bale, S. D., et al. 2016, Space Sci. Rev., 204, 7

Galvin, A. B., Kistler, L. M., Popecki, M. A., et al. 2008, Space Sci. Rev., 136, 437

Gold, R. E., Krimigis, S. M., Hawkins, I. S. E., et al. 1998, Space Sci. Rev., 86, 541

Gómez-Herrero, R., Dresing, N., Klassen, A., et al. 2015, ApJ, 799, 55

Gómez-Herrero, R., Pacheco, D., Kollhoff, A., et al. 2021, A\&A, 656, L3 (SO Cruise Phase SI)

Horbury, T. S., OBrien, H., Carrasco Blazquez, I., et al. 2020, A\&A, 642, A9 Howard, R. A., Moses, J. D., Vourlidas, A., et al. 2008, Space Sci. Rev., 136, 67 Huttunen-Heikinmaa, K., Valtonen, E., \& Laitinen, T. 2005, A\&A, 442, 673 Jokipii, J. R. 1966, ApJ, 146, 480

Khotyaintsev, Yu. V., Graham, D. B., Vaivads, A., et al. 2021, A\&A, 656, A19 (SO Cruise Phase SI) 
Khrabrov, A. V., \& Sonnerup, B. U. 1998, Analysis Methods for MultiSpacecraft Data, 1, 221

Klassen, A., Aurass, H., Klein, K.-L., Hofmann, A., \& Mann, G. 1999, A\&A, 343, 287

Klassen, A., Dresing, N., Gómez-Herrero, R., Heber, B., \& Müller-Mellin, R. 2016, A\&A, 593, A31

Klassen, A., Dresing, N., Gómez-Herrero, R., Heber, B., \& Veronig, A. 2018, A\&A, 614, A61

Klein, K. L., Krucker, S., Trottet, G., \& Hoang, S. 2005, A\&A, 431, 1047

Klein, K.-L., Krucker, S., Lointier, G., \& Kerdraon, A. 2008, A\&A, 486, 589

Kouloumvakos, A., Rouillard, A. P., Wu, Y., et al. 2019, ApJ, 876, 80

Krucker, S., Larson, D. E., Lin, R. P., \& Thompson, B. J. 1999, ApJ, 519, 864

Kühl, P., \& Heber, B. 2019, Space Weather, 17, 84

Kühl, P., Heber, B., Gómez-Herrero, R., et al. 2020, J. Space Weather Space Clim., 10, 53

Laitinen, T., \& Dalla, S. 2019, ApJ, 887, 222

Laitinen, T., Huttunen-Heikinmaa, K., Valtonen, E., \& Dalla, S. 2015, ApJ, 806, 114

Laitinen, T., Kopp, A., Effenberger, F., Dalla, S., \& Marsh, M. S. 2016, A\&A, 591, A18

Lario, D., Raouafi, N. E., Kwon, R.-Y., et al. 2014, ApJ, 797, 8

Lario, D., Kwon, R.-Y., Richardson, I. G., et al. 2017, ApJ, 838, 51

Lemen, J., Title, A., Akin, D., et al. 2011, Sol. Phys., 275, 17

Lepping, R. P., Acũna, M. H., Burlaga, L. F., et al. 1995, Space Sci. Rev., 71, 207

Lin, R. P., Anderson, K. A., Ashford, S., et al. 1995, Space Sci. Rev., 71, 125

Lin, R., Curtis, D., Larson, D., et al. 2008, Space Sci. Rev., 136, 241

Lintunen, J., \& Vainio, R. 2004, A\&A, 420, 343

Maksimovic, M., Bale, S. D., Chust, T., et al. 2020, A\&A, 642, A12

Malandraki, O. E., Marsden, R. G., Lario, D., et al. 2009, ApJ, 704, 469

Marhavilas, P., Malandraki, O., \& Anagnostopoulos, G. 2015, Planet. Space Sci., 117,192

Marsh, M. S., Dalla, S., Kelly, J., \& Laitinen, T. 2013, ApJ, 774, 4

McComas, D., Alexander, N., Angold, N., et al. 2016, Space Sci. Rev., 204, 187

Miteva, R., Klein, K.-L., Kienreich, I., et al. 2014, Sol. Phys., 289, 2601

Müller, D., St. Cyr, O. C., Zouganelis, I., et al. 2020, A\&A, 642, A1

Müller-Mellin, R., Kunow, H., Fleissner, V., et al. 1995, Sol. Phys., 162, 483

Müller-Mellin, R., Böttcher, S., Falenski, J., et al. 2008, Space Sci. Rev., 136, 363

Odstrcil, D. 2003, AdSpR, 32, 497

Odstrčil, D., Smith, Z., \& Dryer, M. 1996, Geophys. Res. Lett., 23, 2521

Ogilvie, K. W., Chornay, D. J., Fritzenreiter, R. J., et al. 1995, Space Sci. Rev., 71,55

Owen, C. J., Bruno, R., Livi, S., et al. 2020, A\&A, 642, A16

Pacheco, D., Agueda, N., Gómez-Herrero, R., \& Aran, A. 2017, J. Space Weather Space Clim., 7, A30

Pacheco, D., Agueda, N., Aran, A., Heber, B., \& Lario, D. 2019, A\&A, 624 A3

Park, J., Innes, D. E., Bucik, R., \& Moon, Y.-J. 2013, ApJ, 779, 184

Posner, A., Bothmer, V., Kunow, H., et al. 1997, Eur. Space Agency Spec. Publ., 415,377

Reinhard, R., \& Wibberenz, G. 1974, Sol. Phys., 36, 473

Richardson, I. G., von Rosenvinge, T. T., Cane, H. V., et al. 2014, Sol. Phys., 289, 3059

Rodríguez-Pacheco, J., Wimmer-Schweingruber, R. F., Mason, G. M., et al. 2020, A\&A, 642, A7

Rouillard, A. P., Sheeley, N. R., Tylka, A., et al. 2012, ApJ, 752, 44

Saiz, A., Evenson, P., Ruffolo, D., \& Bieber, J. W. 2005, ApJ, 626, 1131

Steinvall, K., Khotyaintsev, Yu. V., Cozzani, G., et al. 2021, A\&A, 656, A9 (SO Cruise Phase SI)

Steyn, R., Strauss, D. T., Effenberger, F., \& Pacheco, D. 2020, J. Space Weather Space Clim., 10, 64

Strauss, R. D., Dresing, N., Kollhoff, A., \& Brüdern, M. 2020, ApJ, 897, 24

Thernisien, A. 2011, ApJS, 194, 33

Thernisien, A. F. R., Howard, R. A., \& Vourlidas, A. 2006, ApJ, 652, 763

Torsti, J., Valtonen, E., Lumme, M., et al. 1995, Sol. Phys., 162, 505

Torsti, J., Kocharov, L., Teittinen, M., et al. 1999, J. Geophys. Res., 104, 9903

Vainio, R., Valtonen, E., Heber, B., et al. 2013, J. Space Weather Space Clim., 3 , A12
Vasudevan, G., Shing, L., Mathur, D., et al. 2019, Design and On-orbit Calibration of the Solar Ultraviolet Imager (SUVI) on the GOES-R Series Weather Satellite, 276

von Rosenvinge, T. T., Reames, D. V., Baker, R., et al. 2008, in The High Energy

Telescope for STEREO, ed. C. T, Russell (New York: Springer), 391

Wang, Y., Qin, G., \& Zhang, M. 2012, ApJ, 752, 37

Wibberenz, G., \& Cane, H. V. 2006, ApJ, 650, 1199

Wijsen, N., Aran, A., Sanahuja, B., Pomoell, J., \& Poedts, S. 2020, A\&A, 634, A82

Wijsen, N., Samara, E., Aran, A., et al. 2021, ApJ, 908, L26

Wimmer-Schweingruber, R. F., Janitzek, N. P., Pacheco, D., et al. 2021, A\&A, 656, A22 (SO Cruise Phase SI)

Wraase, S., Heber, B., Böttcher, S., et al. 2018, A\&A, 611, A100

Xu, Z., Guo, J., Wimmer-Schweingruber, R. F., et al. 2020, ApJ, 902, L30

Zhang, M., Qin, G., \& Rassoul, H. 2009, ApJ, 692, 109

Zhang, J., Temmer, M., Gopalswamy, N., et al. 2021, Progr. Earth Planet. Sci., 8,56

Zhao, L., Li, G., Zhang, M., et al. 2019, ApJ, 878, 107

Zhu, B., Liu, Y. D., Kwon, R.-Y., \& Wang, R. 2018, ApJ, 865, 138

1 Institut für Experimentelle und Angewandte Physik, Christian Albrechts-Universität zu Kiel, Kiel, Germany e-mail: kollhoff@physik.uni-kiel.de

2 IRAP, Université Toulouse III - Paul Sabatier, CNRS, CNES, Toulouse, France

3 NASA Goddard Space Flight Center, Heliophysics Science Division, Greenbelt, MD 20771, USA

4 Department of Physics and Astronomy, University of Turku, 20014 Turku, Finland

5 Universidad de Alcalá, Alcalá de Henares, Spain

6 National Observatory of Athens, IAASARS, Metaxa and Vas. Pavlou str., Pedeli 15236, Athens, Greece

7 Department of Astronomy, University of Maryland, College Park, MD 20742, USA

8 NASA/HQ, 300 Hidden Figures Way SW, Washington, DC 20546, USA

9 LESIA, Observatoire de Paris, Université PSL, CNRS, Sorbonne Université, Université de Paris, 5 Place Jules Janssen, 92195 Meudon, France

10 California Institute of Technology, Pasadena, CA, USA

11 University of Central Lancashire, Lancashire, UK

12 Institute for Space Astrophysics and Planetology, INAF, Roma, Italy

13 University of Helsinki, Helsinki, Finland

14 Dep. Física Quàntica i Astrofísica, Institut de Ciències del Cosmos (ICCUB), Universitat de Barcelona (IEEC-UB), Barcelona, Spain

15 Southwest Research Institute, San Antonio, TX 78238, USA

16 Centre for mathematical Plasma-Astrophysics, Department of Mathematics, KU Leuven, Celestijnenlaan 200B, 3001 Leuven, Belgium

17 European Space Astronomy Center, Villanueva de la Cañada, 28692 Madrid, Spain

18 Johns Hopkins University, Applied Physics Laboratory, Laurel, MD, USA

19 Department of Physics and Space Sciences Laboratory, University of California, Berkeley, CA 94720, USA

20 Goddard Planetary Heliophysics Institute, University of Maryland, Baltimore County, Baltimore, MD, USA

21 Department of Physics, Imperial College London, London SW7 2AZ, UK

22 Swedish Institute of Space Physics (IRF), Uppsala 75121, Sweden

23 Radboud Radio Lab, Department of Astrophysics, Radboud University, Nijmegen, The Netherlands

24 Space and Plasma Physics, Department of Physics and Astronomy, Uppsala University, Uppsala 75120, Sweden 